\title{
A continuum model of deformation, transport and irreversible changes in atomic structure
}

\section{in amorphous Lithium-Silicon electrodes}

\author{
Allan F. Bower ${ }^{\mathrm{z}}$, Eric Chason, Pradeep R. Guduru, Brian.W. Sheldon \\ School of Engineering, Brown University, Providence, Rhode Island 02912, USA

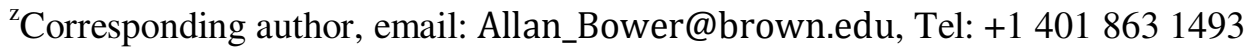

\begin{abstract}
Recent experiments and atomic scale computations indicate that the standard continuum models of diffusion in stressed solids do not accurately describe transport, deformation and stress in Li-Si alloys. We suggest that this is because classical models do not account for the irreversible changes in atomic structure of $\mathrm{Si}$ that are known to occur during a charge-discharge cycle. A more general model of diffusion in an amorphous solid is described, which permits unoccupied Si lattice sites to be created or destroyed. This may occur as a thermally activated process; or as a result of irreversible plastic deformation under stress. The model predicts a range of phenomena observed in experiment that cannot be captured using classical models, including irreversible changes in volume resulting from a chargedischarge cycle, asymmetry between the tensile and compressive yield stress, and a slow evolution in mechanical and electrochemical response over many charge-discharge cycles.
\end{abstract}

\section{Introduction}

There is great interest in using $\mathrm{Si}$ as a high-capacity anode in $\mathrm{Li}$ ion batteries [1]. Its widespread use has been limited by large capacity losses under cyclic charging. Mechanical degradation, driven by the large stresses induced by volume changes in lithiated $\mathrm{Si}$, is believed to be the leading cause of this capacity loss. Managing these stresses through innovative electrode configurations or microstructures is a promising approach to making $\mathrm{Si}$ a practical electrode material [2-7]. This has motivated a large number of recent experimental, theoretical and computational studies of the electrochemical and mechanical response of Li-Si alloys [8].

In particular, recent in-situ observations of deformation in $\mathrm{Si}$ resulting from $\mathrm{Li}$ insertion, as well as controlled measurements of stresses generated in $\mathrm{Si}$ electrodes have revealed that: (i) $\mathrm{Si}$ increases its volume by roughly a factor of three when fully lithiated [9]; (ii) Crystalline silicon transforms to an amorphous structure during the first lithiation cycle, and thereafter remains amorphous $[9,10]$. (iii) A sharp phase boundary develops between crystalline and amorphous Si. The velocity of this phase boundary is a function of the electrode over-potential and is strongly dependent on the orientation of the phase boundary with respect to the underlying crystal [11, 12]; (iv) The mechanical stress-v-strain response of amorphous $\mathrm{Li}-\mathrm{Si}$ is inelastic and irreversible. This was first deduced from experimentally measured stress-v-capacity curves for thin film electrodes, which show reversible linear elastic behavior at small stresses, and an approximately constant flow stress around 1GPa [13]. The elastic modulus and flow stress are functions of composition and the rate of charging $[14,15]$. More recently, rate dependent plastic flow has also been observed directly by nanoindentation tests [16].

Guided by these experiments, a number of models of concurrent transport and deformation in Li-Si alloys have been developed. These have calculated the thermodynamic driving forces for diffusion of $\mathrm{Li}$ in $\mathrm{Li}$ $\mathrm{Si}$, taking into account plasticity and large changes in volume [17]; have determined the influence of stresses on electrochemical reactions [18]; have proposed stress-strain relations for lithiated $\mathrm{Si}$; and have 
treated the phase transformation in lithiated silicon using both traditional Cahn-Hilliard type phase-field methods suitably extended to include plasticity [19], as well as using models in which the diffusion coefficient is concentration dependent [20]. Systematic comparisons between theory and experiment have been used to determine values for material properties of Li-Si in these models [14, 15]. The material models have been used to calculate stress distributions in a number of representative electrode structures, including thin film electrodes [14]; patterned islands [4], and particles [21-23].

Despite these promising results, there are indications from both experiments and atomistic simulations that existing models of $\mathrm{Li}-\mathrm{Si}$ are incomplete. For example, the low flow stress of Li-Si compared to amorphous $\mathrm{Si}$ is somewhat mysterious. Ab-initio atomistic simulations have suggested that the Li insertion process itself reduces the flow stress, since the flow stress of a volume element with a fixed $\mathrm{Li}$ concentration subjected to a mechanical strain exceeds the stresses that develop in a constrained volume element during the Li insertion process itself [24]. Reactive force-field molecular dynamics simulations [25] do not predict this difference between mechanical and insertion induced flow, but suggest that the tensile and compressive flow stresses of lithiated Si differ, which is not predicted by continuum models. In addition, while models are able to capture most qualitative features of experimentally measured stressv-capacity curves, there are features on the experimental data that are not consistent with models, particularly for the first lithiation cycle [14]. The discrepancies have been attributed to capacity loss to SEI formation, but it is possible that other processes play a role. Recent in-situ observations of deformation in amorphous thin film $\mathrm{Si}$ electrodes also revealed the formation of a propagating interface that resembles a phase boundary in these materials [26]. It is not clear what physical mechanism gives rise to this phenomenon, and it would not be predicted by the conventional models of diffusion in a random solid solution. Recent measurements of volume changes in Si electrodes during lithiation also suggest that the change in volume is irreversible [27]. Again, this is not consistent with classical models of transport in solids.

Motivated by these discrepancies, there have been several recent proposals to extend the conventional theory of diffusion in solids to include more complex physical processes that might accompany Li insertion into Si. Brassart and Suo have proposed a 'reactive flow' model for plasticity in amorphous Li$\mathrm{Si}$, in which the usual relationship between concentration and chemical potential is replaced by a rate dependent form, which characterizes a dissipative insertion reaction [28, 29] A thermodynamic consequence of this assumption is that the chemical potential must also influence the flow stress of the material, leading to a strong coupling between mechanical behavior and chemical reactions that is not present in the classical theory of diffusion in stressed solids. Zhang et al [30] have developed a somewhat similar model, in which Li can exist at a point in the Si in two states; a 'reacted' state, and an 'unreacted' state; the transition from one state to the other is dissipative and changes the volume of the alloy. Another model has been further developed by Drozdov [31], who uses a more elaborate description of the solution thermodynamics, as well as strongly concentration dependent diffusion coefficients.

In this paper, we adopt a somewhat different viewpoint. Instead of generalizing the thermodynamics of the Li insertion process, we extend traditional descriptions of transport in solids to take into account the structural changes that occur as a result of Li insertion into Si. Recent models of Li-Si [14, 15, 17-19] are all based on extensions of the Larche-Cahn model of diffusion in solids [32-34], which assumes that transport takes place by discrete atomic hops on a fixed lattice. The original Larche-Cahn model was intended to model only elastic solids, in which the lattice can deform in response to stress, but this deformation is reversible. Larche and Cahn also stipulated that lattice sites can only be created and destroyed at lattice defects such as interfaces, dislocations, or grain boundaries [34]. If this model is applied to $\mathrm{Li}-\mathrm{Si}$, it implies that Li insertion into a stress free material element simply expands or shears the lattice, changing the distance between neighboring atoms and possibly bond angles, but without affecting the underlying lattice structure. The volume of a stress free solid is consequently a unique (but possibly nonlinear) function of the $\mathrm{Li}$ concentration. It is known that deformations resulting from $\mathrm{Li}$ 
insertion are not generally reversible, but extensions of the Larche-Cahn model to Li-Si [14, 17-19] have simply added the possibility of irreversible plastic deformation of the underlying lattice to the original model. The assumption is that plasticity rearranges the underlying lattice but does not change the number of lattice sites, their coordination, or the volume of the solid. In consequence, the volume expansion remains a reversible function of Li concentration. The recent extensions by Zhang et al [30] and Drozdov [31] relax this assumption, but the distinction in these approaches between 'reacted' and 'unreacted' Li in the solid does not have a clear physical interpretation.

Here, we suggest that $\mathrm{Li}$ insertion into $\mathrm{Si}$ is accompanied by an irreversible, dissipative transformation of the underlying Si network, and that models of Li-Si based on the Larche-Cahn theory of transport in solids must be modified in order to take this into account. The transformation of crystalline Si to an amorphous structure upon Li insertion is clear evidence that irreversible changes in Si structure occur at least during the first lithiation cycle. There is good reason to believe that similar atomic-scale rearrangements must occur even in amorphous $\mathrm{Si}$. These changes in $\mathrm{Si}$ structure have several consequences. Firstly, the free energy of stress free Li-Si is no longer only a function of concentration, but also of the atomic structure. At the same time, the atomic structure is not uniquely determined by composition, but is dependent on the history of electrochemical and mechanical loading. This is the case for all solids, of course, but it is usually assumed that the atomic structure will adopt its minimum energy configuration over time-scales that are very short compared to those associated with transport, and that the structural contribution to free energy is negligible to the chemical binding energy. At least the first of these, and quite possibly the second, is not true for Li-Si. Secondly, transport in amorphous Si is known to be highly sensitive to its atomic structure. The Li diffusion coefficient in Li-Si is therefore likely to evolve with its atomic structure and may be history dependent. Thirdly, changes in the Li-Si structure are likely to result in a volume change. This implies that stresses will play a role in driving the structural changes, and in turn, the structural changes will alter the mechanical behavior of Li-Si alloys. This provides a mechanistic explanation for the coupling between the Li insertion reaction that is captured by recent 'reactive flow' models.

Our goal in this paper is therefore to extend the Larche-Cahn model of diffusion in stressed solids to account for irreversible changes in the atomic structure that occur concurrently with diffusion. The extensions are relatively minor. Like Larche and Cahn, we assume that diffusion in Li-Si takes place by discrete atomic jumps on a fixed (but randomly structured) lattice network, in which some sites are unoccupied. The lattice structure can deform under stress. We merely extend this picture by allowing the number of potential sites that can be occupied by Si in the solid to vary. New unoccupied sites can be nucleated by a thermally activated process, driven by the appropriate thermodynamic driving force (which includes a contribution from stress). If the number of vacant sites exceeds the equilibrium concentration, the same process allows excess sites to be annihilated. In addition, we assume that plastic shear deformation induced by mechanical stresses that exceed the flow stress will nucleate additional vacancies, by disrupting the underlying atomic structure.

We describe this idealization in more detail in the following section; and outline a set of thermodynamically consistent constitutive equations that might characterize the processes of interest. The qualitative predictions of this model are discussed and compared with previous models in Section 3. Conclusions, and possible future extensions of the model are discussed in Section 4.

\section{Model.}

Our model follows closely the description of equilibrium and transport in multi-component elastic solids proposed by Larche and Cahn [32, 33], with two important extensions. In the original Larche Cahn model, diffusion takes place by exchange of atoms (or atom/vacancy exchange) on a fixed lattice. The 
lattice can be distorted by stresses, but the deformation is reversible (elastic) and preserves lattice sites. Here, we extend this model to account for irreversible distortions of the lattice, and also to allow lattice sites to be created or destroyed by a thermally activated process.

To make these ideas specific, consider a volume element which is large compared to the atomic spacing, but small enough to regard its composition and structure as uniform. Our idealization is illustrated in Fig. 1. The volume element has five features:

(i) As in the original Larche-Cahn model, we assume that at any instant of time, the volume element contains a finite set of atomic sites (which would be the lattice sites in a crystalline material, but since Li$\mathrm{Si}$ is amorphous we prefer not to use the word 'lattice'). These sites may be occupied by an atom, or may be vacant. Since $\mathrm{Li}$ is an interstitial in $\mathrm{Si}-\mathrm{Li}$ alloys, we assume that $\mathrm{Li}$ and $\mathrm{Si}$ occupy separate sites. In Fig 1, red circles denote Si sites that are occupied by $\mathrm{Si}$ atoms, while open circles represent sites which are available to accommodate additional $\mathrm{Si}$ atoms. The Si sites form an amorphous network. We assume that Li atoms (shown as small blue circles in Fig 1) can either occupy interstitial sites (which are denoted by black dots in Fig 1), or alternatively can occupy vacant Si sites. We assume that the number of interstitial $\mathrm{Li}$ sites in a volume element is proportional to the number of $\mathrm{Si}$ atoms. In addition, if a vacant $\mathrm{Si}$ site is occupied by an $\mathrm{Li}$ atom, we assume that it is no longer available to $\mathrm{Si}$ atoms.

(ii) $\mathrm{Si}$ and Li atoms diffuse by jumps into a vacant atomic site, or by mutual exchange. This preserves the network structure, but allows $\mathrm{Si}$ or $\mathrm{Li}$ atoms and vacancies to leave the volume element, resulting in a change in composition.

(iii) We assume that vacant Si sites can be created or destroyed by a thermally activated process. In a crystal lattice, vacancies would be nucleated as a result of dislocation climb. The corresponding mechanism for nucleating free volume in an amorphous solid is less well understood, but free volume changes have been directly observed in amorphous glasses and polymers [35], and many models of amorphous solids include the free volume as a material state variable [36] [37]. We adopt a similar approach here.

(iv) If the solid is subjected to stress, at short time scales the volume element deforms elastically, which results in a small, reversible change in the spacing and angles between atomic sites. We assume that elastic deformations are small enough to be described using conventional linear elastic strain rate measures.

(v) Finally, if the stresses applied to the solid are sufficiently large, and are applied for a sufficiently long period of time, the volume element can deform plastically. At the microscopic level, this involves a local rearrangement of the amorphous Si network. This is an irreversible, dissipative process resembling viscoplastic flow in amorphous glasses and metals. The deformation is primarily a volume preserving shear involving a local rearrangement of the Si network without changing its overall structure. Unlike metal plasticity, however, viscoplastic flow in amorphous materials may generate free volume [36, 37]. We model this process by assuming that vacant $\mathrm{Si}$ sites are generated at a rate that depends on the magnitude of the plastic strain rate.

We will characterize the composition and structure of the solid as follows. At some instant of time, an element of material with volume $V$ (such as that illustrated schematically in Fig 1) contains $N_{L i}$ mols of Li atoms; $N_{S i}$ mols of Si atoms, and $N_{V}$ mols of unoccupied Si sites. We suppose that $\beta N_{L i}$ atoms reside in $\mathrm{Si}$ sites (which are then no longer available to $\mathrm{Si}$ atoms); while $(1-\beta) N_{L i} \mathrm{Li}$ atoms occupy 
interstitial sites, where $0<\beta<1$ is a material constant. Note that the total number of available Si sites in the amorphous network follows as

$$
N_{A}=N_{S i}+N_{V}+\beta N_{L i}
$$

If the number of $\mathrm{Si}$ sites $N_{A}$ is constant, and $\beta$ is selected appropriately, this description will reduce to standard network binary solution models. For example, selecting $\beta=1$, and taking the limit $N_{V}=0$ produces a binary substitutional solution in which both $\mathrm{Li}$ and $\mathrm{Si}$ share the same network sites. Similarly, selecting $\beta=0$ and taking $N_{V}=0$ produces a binary solution in which Si atoms occupy fixed lattice sites, and Li atoms occupy interstitial sites in the Si network. A material with nonzero values of $N_{V}$ can be interpreted as a ternary solid solution in which vacancies are one constituent. The calculations here generalize the standard models by allowing $\beta$ to have values between 0 and 1 . In addition, in our description $N_{A}$ is not constant, but may increase and decrease as vacant $\mathrm{Si}$ sites are nucleated or annihilated. These extensions are both speculative: other descriptions are possible, and may prove necessary as more details of the atomic scale processes of deformation and transport in Li-Si emerge.

If eq. (1) is divided by the volume of the material element, it can be expressed in terms of the spatial concentrations $c_{S i}=N_{S i} / V, c_{L i}=N_{L i} / V, c_{V}=N_{V} / V, c_{A}=N_{A} / V$ as

$$
\beta c_{L i}+c_{S i}+c_{V}=c_{A}
$$

Any microscopic processes that occur in the solid must obey this constraint. It is helpful to think of $c_{S i}, c_{L i}, c_{A}$ as independent variables, and regard $c_{V}$ as a dependent variable. In this picture, changing $c_{L i}$, with $c_{S i}, c_{A}$ fixed, can be interpreted as a change in composition resulting from $\mathrm{Li}$ atoms leaving or entering the volume element by exchanging with vacant sites (some Li atoms are accommodated in interstitial sites; others in vacant $\mathrm{Si}$ sites). Similarly, changing $c_{S i}$ represents transport of $\mathrm{Si}$ atoms into the material element, while increasing $c_{A}$ with $c_{L i}, c_{S i}$ fixed represents the creation of vacancies, or free volume in the Si network.

The motion of the material is characterized by the velocity $\mathbf{v}$ of the Si atomic sites with respect to a stationary observer. In addition, $\mathrm{Li}$, and possibly also $\mathrm{Si}$ atoms, may transport through the network, by mutual exchange, or by exchange with vacancies. We characterize the diffusion of atoms relative to the underlying network structure by vectors $\mathbf{j}_{L i}, \mathbf{j}_{S i}$ which quantify the molar flux of each species crossing a material plane per unit time. To make this specific, define an element of area that convects with the Si network through $d A \mathbf{n}=d \mathbf{y}_{1} \times d \mathbf{y}_{2}$, where $d \mathbf{y}_{1}, d \mathbf{y}_{2}$ are two infinitesimal vectors (e.g. vectors connecting two neighboring sites) that convect with the atomic network. Then $q_{L i}=d A \mathbf{j}_{L i} \cdot \mathbf{n}$ is the number of mols per second of Li crossing this area element.

The concentrations and fluxes of $\mathrm{Li}$ and $\mathrm{Si}$ are related by conservation laws

$$
\frac{D c_{L i}}{D t}+c_{L i} \nabla \cdot \mathbf{v}+\nabla \cdot \mathbf{j}_{L i}=0 \quad \frac{D c_{S i}}{D t}+c_{S i} \nabla \cdot \mathbf{v}+\nabla \cdot \mathbf{j}_{S i}=0
$$

The concentration of $\mathrm{Si}$ atomic sites evolves according to

$$
\frac{D c_{A}}{D t}+c_{A} \nabla \cdot \mathbf{v}=\frac{D n_{A}}{D t}
$$

where $D n_{A} / D t$ is the molar density of vacant sites nucleated per unit time. Here $D / D t$ denotes a material time derivative - i.e. the time derivative of a quantity taken by an observer who convects with the atomic network with velocity v. The gradient operator $\nabla$ denotes a derivative taken with respect to position in space. All material quantities, including the concentrations, are assumed to convect with the network of atomic sites. Consequently, the spatial time derivative of concentration (i.e. the time derivative at a fixed point in space) is related to the material derivative by 


$$
\frac{d c}{d t}=\frac{D c}{D t}+\nabla c \cdot \mathbf{v}
$$

where $c$ may have subscripts $\mathrm{Li}, \mathrm{Si}$, or A. A similar expression holds for all time derivatives that appear in equations to follow. Equation (5) differs from models of transport in fluids [38], which usually use the mean particle velocity or the mass average velocity in place of the lattice velocity $\mathbf{v}$ to relate spatial to material derivatives. The difference arises because the atoms in fluids do not have preferred sites, and consequently models of fluids do not include the concept of a 'vacancy.'

The solid is loaded by tractions $\mathbf{t}$ on its external surface, and a body force $\mathbf{b}$ per unit mass. Inertia is neglected. The loading induces a state of 'true' stress (force per unit deformed area) in the solid $\boldsymbol{\sigma}$, which satisfies the static equilibrium equation $\nabla \cdot \boldsymbol{\sigma}+m \mathbf{b}=0$ and boundary conditions $\boldsymbol{\sigma} \cdot \mathbf{n}=\mathbf{t}$ on the exterior surface.

\subsection{Deformation}

The deformation of the material is characterized by the velocity gradient $\mathbf{L}=\nabla \mathbf{v}$, which can be decomposed into a symmetric 'strain rate' and a skew-symmetric 'spin' as

$$
\dot{\boldsymbol{\varepsilon}}=\left(\nabla \mathbf{v}+\nabla \mathbf{v}^{T}\right) / 2 \quad \mathbf{W}=\left(\nabla \mathbf{v}-\nabla \mathbf{v}^{T}\right) / 2
$$

The strain rate is a consequence of four microscopic processes that take place within a volume element: (i) The composition of the volume element may change as $\mathrm{Li}, \mathrm{Si}$ and vacancies flow in or out of the cell;

(ii) New atomic sites can be created or deleted, which results in a volume change; (iii) The solid may deform by plastic shearing of the atomic network, which is volume preserving; (iv) the solid may deform elastically, stretching or changing angles between bonds. We describe this by decomposing the total strain rate into contributions from changes in composition; plastic shearing; and elastic deformation as

$$
\dot{\boldsymbol{\varepsilon}}=\dot{\boldsymbol{\varepsilon}}^{e}+\dot{\boldsymbol{\varepsilon}}^{p}+\dot{\boldsymbol{\varepsilon}}^{c}
$$

The strain resulting from changes in composition can be visualized as follows. We consider a volume of material $V$, which is free of stress and has uniform composition. Within this volume element, we suppose that $\mathrm{Li}$ and $\mathrm{Si}$ atoms have partial molar volumes $\Omega_{L i}, \Omega_{S i}$, while the vacant $\mathrm{Si}$ sites (which are not occupied by either $\mathrm{Si}$ or $\mathrm{Li}$ ) have a partial molar volume $\Omega_{V}$. For simplicity, we assume that $\left\{\Omega_{L i}, \Omega_{S i}, \Omega_{V}\right\}$ are independent of composition, although this need not necessarily be the case (strictly, $\left\{\Omega_{L i}, \Omega_{S i}, \Omega_{V}\right\}$ are the rate of change of volume with an infinitesimal change in $\left\{N_{L i}, N_{S i}, N_{V}\right\}$, respectively). It follows that the total volume of the stress free material element is given by

$$
V=N_{L i} \Omega_{L i}+N_{S i} \Omega_{S i}+N_{V} \Omega_{V}=N_{L i}\left(\Omega_{L i}-\beta \Omega_{V}\right)+N_{S i}\left(\Omega_{S i}-\Omega_{V}\right)+N_{A} \Omega_{V}
$$

Dividing by $V$ yields

$$
c_{L i}\left(\Omega_{L i}-\beta \Omega_{V}\right)+c_{S i}\left(\Omega_{S i}-\Omega_{V}\right)+c_{A} \Omega_{V}=1
$$

We can now change the composition of the material, either by placing $d N_{L i}, d N_{S i}$ mols of Li or Si into vacant atomic sites, or by nucleating $d N_{A}$ mols of new vacant sites within in the volume element. As a result, its volume changes by an amount

$$
d N_{L i}\left(\Omega_{L i}-\beta \Omega_{V}\right)+d N_{S i}\left(\Omega_{S i}-\Omega_{V}\right)+d N_{A} \Omega_{V}=d V
$$

Changes in composition therefore result in a volumetric strain rate

$$
\frac{1}{V} \frac{d V}{d t}=\frac{1}{V} \frac{d N_{L i}}{d t}\left(\Omega_{L i}-\beta \Omega_{V}\right)+\frac{1}{V} \frac{d N_{S i}}{d t}\left(\Omega_{S i}-\Omega_{V}\right)+\frac{1}{V} \frac{d N_{A}}{d t} \Omega_{V}
$$

We can therefore express the strain rate resulting from changes in composition as 


$$
\dot{\boldsymbol{\varepsilon}}^{c}=\left(-\left(\Omega_{L i}-\beta \Omega_{V}\right) \nabla \cdot \dot{\mathbf{j}}_{L i}-\left(\Omega_{S i}-\Omega_{V}\right) \nabla \cdot \dot{\mathbf{j}}_{S i}+\Omega_{V} \frac{D n_{A}}{D t}\right) \frac{\mathbf{I}}{3}
$$

where $D n_{A} / D t$ is the number of mols of $\mathrm{Si}$ atomic sites nucleated per unit volume per second, and $\mathbf{I}$ is the identity tensor.

We assume that two processes contribute to the vacancy nucleation rate $D n_{A} / D t$ : firstly, they may nucleate spontaneously by a thermally activated process, so as to reduce the total free energy of the solid; secondly, they may be nucleated as a by-product of plastic deformation, which rearranges the Si network. Thus

$$
\frac{D n_{A}}{D t}=v^{P}\left(\dot{\boldsymbol{\varepsilon}}^{p}\right)+v^{T}\left(\mu_{A}\right)
$$

where $\quad \boldsymbol{v}^{P}\left(\dot{\boldsymbol{\varepsilon}}^{p}\right)$ is a function representing vacancy production by plastic flow; $\boldsymbol{v}^{T}\left(\mu_{A}\right)$ quantifies thermally activated vacancy nucleation, and $\mu_{A}$ is the vacancy chemical potential. These nucleation rates, as well as the elastic and plastic strain rates $\dot{\boldsymbol{\varepsilon}}^{e}, \dot{\boldsymbol{\varepsilon}}^{p}$ must be related to stress and composition by constitutive equations. Thermodynamic restrictions provide some broad guidelines for the structure of these constitutive equations.

\subsection{Thermodynamics}

The relevant thermodynamic driving forces for transport, free volume nucleation, and deformation are most conveniently calculated from the specific Gibbs free energy (i.e. the Gibbs free energy per unit mass). We assume that the specific free energy $g$ depends only on composition (through concentrations $c_{L i}, c_{S i}$ and the molar density of Si sites $c_{A}$ ) and stress. A lengthy calculation that will not be reproduced here shows that the specific Gibbs free energy of the system of interest can be decomposed additively into a stress-free contribution, a strain energy per unit mass, and the usual contribution from pressure

$$
g=g_{0}\left(c_{L i}, c_{S i}, c_{A}\right)+\frac{1}{m}\left\{c_{L i}\left(\Omega_{L i}-\beta \Omega_{V}\right)+c_{S i}\left(\Omega_{S i}-\Omega_{V}\right)+c_{A} \Omega_{V}\right\}\left\{\phi_{e}(\boldsymbol{\sigma})-\frac{1}{3} \operatorname{tr}(\boldsymbol{\sigma})\right\}
$$

where $g_{0}$ is the specific free energy of a stress free material, $\phi_{e}$ is the strain energy density per unit volume; $\operatorname{tr}(\boldsymbol{\sigma})$ denotes the hydrostatic stress, and $m$ is the mass density. The mass density depends on composition, and is related to the molar masses $m_{L i}$ and $m_{S i}$ of Li and Si by

$$
m=m_{L i} c_{L i}+m_{S i} c_{S i}
$$

Equation (14) may look somewhat perverse in light of (9), but has been written in this form so its derivatives with respect to concentration can be computed. For small elastic strains, the elastic strain energy density can be approximated as

$$
\phi_{e}=\frac{1}{4 \mu}\left(\sigma_{i j} \sigma_{i j}-\frac{v}{1+v}\left(\sigma_{k k}\right)^{2}\right)
$$

where $\mu$ is the shear modulus and $v$ is the Poisson's ratio of the material. Both $\mu$ and $v$ could be functions of composition. The chemical potentials of $\mathrm{Li}$ and $\mathrm{Si}$ are given by the usual expressions relating the total Gibbs free energy $G$ and the number of mols of each species 


$$
\begin{aligned}
& \mu_{L i}=\frac{\partial G}{\partial N_{L i}}=\frac{\partial m g}{\partial c_{L i}}=\frac{\partial m g_{0}}{\partial c_{L i}}+\left(\Omega_{L i}-\beta \Omega_{V}\right)\left\{\phi_{e}(\boldsymbol{\sigma})-\frac{1}{3} \operatorname{tr}(\boldsymbol{\sigma})\right\} \\
& \mu_{S i}=\frac{\partial G}{\partial N_{S i}}=\frac{\partial m g}{\partial c_{S i}}=\frac{\partial m g_{0}}{\partial c_{S i}}+\left(\Omega_{S i}-\Omega_{V}\right)\left\{\phi_{e}(\boldsymbol{\sigma})-\frac{1}{3} \operatorname{tr}(\boldsymbol{\sigma})\right\}
\end{aligned}
$$

Similarly, the thermodynamic driving force for nucleation or annihilation of vacant atomic sites is

$$
\mu_{A}=\frac{\partial G}{\partial N_{A}}=\frac{\partial m g}{\partial c_{A}}=\frac{\partial m g_{0}}{\partial c_{A}}+\Omega_{V}\left\{\phi_{e}(\boldsymbol{\sigma})-\frac{1}{3} \operatorname{tr}(\boldsymbol{\sigma})\right\}
$$

It is worth noting that these expressions reduce to those previously derived for transport in both solids and fluids in appropriate limits. For the limit considered by Larche and Cahn, the lattice remains fixed, and atomic sites cannot be created or annihilated. The chemical potentials in (17) reduce to Larche-Cahn's results, with the exception of the term involving strain energy density, which was later added by Wu [39]. In the limiting case where vacancies can be nucleated freely, the expressions also reduce to those commonly used in modeling transport in fluids. To show this, note that to ensure that the Gibbs free energy is extensive, the specific free energy must satisfy $g\left(N_{L i}, N_{S i}, N_{V}, \boldsymbol{\sigma}\right)=g\left(\lambda N_{L i}, \lambda N_{S i}, \lambda N_{V}, \boldsymbol{\sigma}\right)$. The terms involving stress in equation (14) satisfy this condition directly, while the stress free part of the free energy must be a homogeneous function of degree zero, which by Euler's theorem requires that

$$
c_{L i} \frac{\partial g_{0}}{\partial c_{L i}}+c_{S i} \frac{\partial g_{0}}{\partial c_{S i}}+c_{A} \frac{\partial g_{0}}{\partial c_{A}}=0
$$

It then follows from eqs (9), (18), (19), (17) that

$$
c_{L i} \mu_{L i}+c_{S i} \mu_{S i}+c_{A} \mu_{A}=m g
$$

Taking the gradient of both sides of this expression and simplifying the result using (17), (18) yields the gradient Gibbs condition

$$
c_{L i} \nabla \mu_{L i}+c_{S i} \nabla \mu_{S i}+c_{A} \nabla \mu_{A}=\left(\frac{\partial \phi_{e}}{\partial \boldsymbol{\sigma}} \nabla \boldsymbol{\sigma}-\nabla \frac{\operatorname{tr}(\boldsymbol{\sigma})}{3}\right)
$$

If vacancies are in thermodynamic equilibrium $\nabla \mu_{A}=0$. In addition, in most models of fluids the elastic strain energy is neglected. In this case (21) reduces to the standard Gibbs-Duhem relation that provides the starting point for modeling transport in fluids [38]. Note, however, that the chemical potentials for Li and Si satisfy the classical Gibbs-Duhem condition only if the vacancies are in equilibrium, whereas (17), (18) and (21) are general.

The stress-free specific Gibbs free energy $g_{0}$ must be determined experimentally or by ab-initio simulations. Here, we will assume that the free energy can be fit by a function of the form

$$
\begin{aligned}
& g_{0}=g_{c}+g_{V} \\
& g_{c}=\sum_{n=1}^{N} \frac{\Lambda_{n} r c_{S i}}{\left(m_{S i} c_{S i}+m_{L i} c_{L i}\right)}\left(\frac{c_{L i}}{r c_{S i}}\right)^{n}+\frac{R T\left(r c_{S i}-c_{L i}\right)}{\left(m_{S i} c_{S i}+m_{L i} c_{L i}\right)} \log \left(1-\frac{c_{L i}}{r c_{S i}}\right)+\frac{R T c_{L i}}{\left(m_{S i} c_{S i}+m_{L i} c_{L i}\right)} \log \left(\frac{c_{L i}}{r c_{S i}}\right) \\
& g_{V}=\varepsilon_{V} \frac{\left(c_{A}-c_{S i}-\beta c_{L i}\right)}{\left(m_{S i} c_{S i}+m_{L i} c_{L i}\right)}+R T \frac{c_{A}-c_{S i}+\beta c_{L i}}{\left(m_{S i} c_{S i}+m_{L i} c_{L i}\right)} \log \left(1-\frac{c_{S i}+\beta c_{L i}}{c_{A}}\right)+\frac{R T\left(c_{S i}+\beta c_{L i}\right)}{\left(m_{S i} c_{S i}+m_{L i} c_{L i}\right)} \log \left(\frac{c_{S i}+\beta c_{L i}}{c_{A}}\right)
\end{aligned}
$$

Here $R$ is the universal gas constant, and $T$ is temperature. The two contributions $g_{c}, g_{V}$ account for chemical bonding and mixing of $\mathrm{Li}$ and $\mathrm{Si}$, and the effects of vacancies or free volume in the Si network, respectively. The first term in the expression for $g_{c}$ represents non-ideal solution thermodynamics, and can be interpreted loosely as a generalized quasi-chemical approximation. The coefficients $\Lambda_{n}$ were fit to ab-initio computations from ref [40] and compared with experimental measurements in ref [14]. The second term represents the entropic contribution of unoccupied Li sites. In each term $r=3.75$ is the 
number of $\mathrm{Li}$ sites per $\mathrm{Si}$ atom. The contribution to the specific free energy from $g_{V}$ accounts for the effects of vacant $\mathrm{Si}$ atomic sites. In this expression $\varepsilon_{V}$ is the formation energy of one mol of unoccupied $\mathrm{Si}$ sites, and $0<\beta<1$ is the fraction of Li that occupies vacant Si sites. Equation (22), and particularly the parameter $\beta$, is somewhat speculative: the entropic terms are taken from the usual expressions for the entropy of vacancies on a regular lattice (recall that setting $\beta=1$ would represent a substitutional binary alloy, while $\beta=0$ is an interstitial alloy), and may not apply to Li-Si. We use (22) in the hope that it captures the essential role of disorder and solution thermodynamics in Li-Si, but with the expectation that future more careful studies will improve it.

Plastic deformation, nucleation of atomic sites, and transport are irreversible processes and play no role in the free energy in the chemical potential. To satisfy the second law, constitutive equations describing these processes must ensure that plastic strain rate, lattice site production rate, and transport always generate heat. This requires that

$$
\boldsymbol{\sigma}: \dot{\boldsymbol{\varepsilon}}^{p}-\mu_{A} \boldsymbol{v}^{p}\left(\dot{\boldsymbol{\varepsilon}}^{p}\right) \geq 0 \quad \mu_{A} v^{T}\left(\mu_{A}\right) \leq 0 \quad \mathbf{j}_{L i} \cdot \nabla \mu_{L i}+\mathbf{j}_{S i} \cdot \nabla \mu_{S i} \leq 0
$$

The consequences of these restrictions are explored in more detail in the next section.

\subsection{Constitutive equations}

It remains to specify constitutive equations that relate the elastic and plastic strain rates $\dot{\boldsymbol{\varepsilon}}^{e}, \dot{\boldsymbol{\varepsilon}}^{p}$, the fluxes $\mathbf{j}_{L i}, \mathbf{j}_{S i}$ and the rate of vacancy nucleation $D n_{A} / D t$ to the stress, stress rate, and chemical potentials of the various species. For an isotropic solid with strain energy density given by equation (16) one can show [41] that the elastic strain rate is related to stress by

$$
\dot{\boldsymbol{\varepsilon}}^{e}=\frac{1}{2 \mu}\left(\stackrel{\nabla}{\boldsymbol{\sigma}}-\frac{v}{1+\nu} \operatorname{trace}(\boldsymbol{\sigma}) \mathbf{I}\right)
$$

where

$$
\stackrel{\nabla}{\boldsymbol{\sigma}}=\frac{D \boldsymbol{\sigma}}{D t}-\mathbf{W} \boldsymbol{\sigma}+\boldsymbol{\sigma} \mathbf{W}
$$

is the Jaumann stress rate and $\mathbf{W}$ is the material spin defined in eq. (6). It is important to note that eqs (24), (25) are valid only if the modulus $\mu$ in (24) greatly exceeds the magnitude of the stress (quantified, e.g. by the greatest principal stress). If the stress becomes comparable to the modulus, eq. (25) contains some additional terms, and the linear elastic relation between stress rate and strain rate in (24) may no longer be accurate. Equation (24) remains valid even if strains resulting from changes in composition, vacancy nucleation, or plasticity are arbitrarily large, however.

We relate the plastic strain rate to stress using a modified non-Newtonian power-law viscous relation commonly used to model flow in amorphous materials near the glass transition temperature

$$
\dot{\boldsymbol{\varepsilon}}^{p}=\dot{\varepsilon}_{0}\left(\frac{\sigma_{e}}{\sigma_{f}\left(\mu_{A}\right)}\right)^{m} \frac{3}{2} \frac{\mathbf{S}}{\sigma_{e}} \quad \mathbf{S}=\boldsymbol{\sigma}-\frac{1}{3} \operatorname{tr}(\boldsymbol{\sigma}) \quad \sigma_{e}=\sqrt{\frac{3}{2} \mathbf{S}: \mathbf{S}}
$$

Here, $\dot{\varepsilon}_{0}$ is a temperature dependent material constant, and $\sigma_{f}\left(\mu_{A}\right)$ is a characteristic flow stress, which must depend on the Si vacancy chemical potential in order to satisfy the first of (23), $\mathbf{S}$ denotes the deviatoric stress and $\mathbf{S}: \mathbf{S}$ is a contracted product. The plastic constitutive law must also specify the rate of nucleation of atomic sites as a function of the plastic strain rate $v^{p}\left(\dot{\boldsymbol{\varepsilon}}^{p}\right)$. If the material is isotropic, then 
$v^{p}\left(\dot{\boldsymbol{\varepsilon}}^{p}\right)$ is a function of the magnitude of the plastic strain rate tensor $\dot{\varepsilon}_{e}=\sqrt{2 \dot{\boldsymbol{\varepsilon}}: \dot{\boldsymbol{\varepsilon}} / 3}$, in which case the first of eq (23) can be re-written as

$$
\dot{\varepsilon}_{e} \sigma_{f}\left(\mu_{A}\right)\left(\frac{\dot{\varepsilon}_{e}}{\dot{\varepsilon}_{0}}\right)^{1 / m}-\mu_{A} v^{p}\left(\dot{\varepsilon}_{e}\right) \geq 0
$$

We can satisfy this condition for all plastic strain rates and vacancy chemical potentials by selecting

$$
v^{p}\left(\dot{\varepsilon}_{e}\right)=v_{0}^{p}\left(\frac{\dot{\varepsilon}_{e}}{\dot{\varepsilon}_{0}}\right)^{(m+1) / m} \quad \sigma_{f}\left(\mu_{A}\right)=\sigma_{0}+\mu_{A} \frac{v_{0}^{p}}{\dot{\varepsilon}_{0}}
$$

Here, $\sigma_{0}$ is a flow stress and $v_{0}$ is a characteristic vacancy nucleation rate. Both $\sigma_{0}$ and $v_{0}$ could depend on composition and temperature. It is helpful to note that this choice also ensures that the sum of the plastic strain rate and the volumetric strain rate resulting from plastic strain driven vacancy nucleation satisfies

$$
\dot{\boldsymbol{\varepsilon}}^{p}+\frac{1}{3} \Omega_{V} v^{p}\left(\dot{\boldsymbol{\varepsilon}}^{p}\right) \mathbf{I}=\Omega_{V} v_{0}^{p}\left(\frac{\sigma_{e}}{\sigma_{f}\left(\mu_{A}\right)}\right)^{m+1} \mathbf{I}+\dot{\varepsilon}_{0}\left(\frac{\sigma_{e}}{\sigma_{f}\left(\mu_{A}\right)}\right)^{m} \frac{3}{2} \frac{\mathbf{S}}{\sigma_{e}} \approx \frac{\partial}{\partial \boldsymbol{\sigma}}\left\{\frac{\dot{\varepsilon}_{0}}{m+1} \frac{\sigma_{e}^{m+1}}{\sigma_{f}^{m}}\right\}
$$

where only the strain energy density term in eq (18) was neglected to obtain the last result. This property implies that the strain rate is the derivative of a viscoplastic potential with respect to stress. This is a desirable property since solutions to boundary value problems for such materials are known to be wellposed and stable.

Diffusion equations consistent with the third of (23) have the form

$$
\mathbf{j}_{L i}=-L_{L i L i}\left(\nabla \mu_{L i}-m_{L i} \mathbf{b}\right)-L_{S i L i}\left(\nabla \mu_{S i}-m_{S i} \mathbf{b}\right) \quad \mathbf{j}_{S i}=-L_{S i S i}\left(\nabla \mu_{S i}-m_{S i} \mathbf{b}\right)-L_{S i L i}\left(\nabla \mu_{L i}-m_{L i} \mathbf{b}\right)
$$

where $L_{A B}$ are phenomenological diffusion coefficients, which must satisfy the Onsager symmetry condition $L_{A B}=L_{B A}$, and $\mathbf{b}$ is the external body force per unit mass acting on the solid. Body forces are usually negligible in practice: they are included in eq. (30) to show that the driving force reduces to eq (1) of Christensen and Newman [42] under appropriate conditions. Their expression for the driving force for diffusion includes a pressure gradient term proportional to the molar mass of the species. To understand the origin of this term, note that if inertia and shear stresses are negligible one can set $\mathbf{b}=-(\nabla \cdot \boldsymbol{\sigma}) / m \approx \nabla p / m$, yielding (under isothermal conditions) eq. (1) of ref [42]. This is a good approximation for a fluid, but is not generally valid for a solid. Curiously, previous treatments [43] of diffusion in solids have assumed that body forces act only on the atomic network, and not the diffusing species themselves, and therefore do not include the body force term in (30). These models therefore do not predict correctly the effects of gravity on a solid solution consisting of two species with different molar masses. These concerns are unimportant for Li-Si electrodes where body forces are negligible, but suggest that some old questions [44] concerning the consistency of treatments of diffusion in solids and liquids may not yet have been fully resolved .

One of several different expressions could be adopted for the phenomenological coefficients in terms of tracer diffusion coefficients. For example, the Manning relations [45] yield 


$$
\begin{aligned}
& L_{L i L i}=\frac{c_{L i} D_{L i}}{R T}\left(1+\frac{\left(1-f_{0}\right) c_{L i} D_{L i}}{f_{0}\left(c_{L i} D_{L i}+c_{S i} D_{S i}\right)}\right) L_{S i S i}=\frac{c_{S i} D_{S i}}{R T}\left(1+\frac{\left(1-f_{0}\right) c_{S i} D_{S i}}{f_{0}\left(c_{L i} D_{L i}+c_{S i} D_{S i}\right)}\right) \\
& L_{S i L i}=L_{L i S i}=\frac{\left(1-f_{0}\right) c_{S i} D_{S i} c_{L i} D_{L i}}{R T f_{0}\left(c_{L i} D_{L i}+c_{S i} D_{S i}\right)}
\end{aligned}
$$

where $f_{0}$ is the geometric tracer correlation factor for the lattice, and $D_{L i}, D_{S i}$ are the tracer diffusion coefficients of $\mathrm{Li}$ and $\mathrm{Si}$, which are functions of temperature. The Manning relations were originally derived for a random alloy in which the two species diffuse on the same lattice, but have since been shown to hold more generally. Correlation factors depend on the crystal structure and the mechanism of diffusion - values are tabulated for simple structures in ref [45] (eg $f_{0}=1$ for random walk; $f_{0}=0.78$ for an fcc lattice). For more complex systems the correlation factor can exceed 1, and may depend on temperature. In most prior models of $\mathrm{Li}-\mathrm{Si}, \mathrm{Si}$ has been assumed to be immobile: this limit can be recovered by setting $f_{0}=1, D_{S i}=0$, which then yields a classical diffusion equation for Li. Whether (31) applies to amorphous Li-Si is not clear. Diffusion in amorphous solids involves more complex atomicscale processes than simple vacancy/atom exchanges and consequently the diffusion coefficient in amorphous $\mathrm{Si}$ is sensitive to its structure [35,46]. Atomistic studies are beginning to reveal some of these processes but a generally accepted model does not exist at this time.

Thermally activated vacancy nucleation in the Si lattice may be modeled by a similar power-law constitutive equation of the form

$$
v^{T}\left(\mu_{A}\right)=-v_{0}^{T}\left(\frac{\left|\mu_{A}\right|}{R T}\right)^{n-1} \frac{\mu_{A}}{R T}
$$

Here, $v_{0}^{T}$ and $n$ are material parameters.

For practical calculations, it is convenient to combine some of the governing equations listed in the preceding sections into a simpler form. The chemical potentials can be reduced to

$$
\begin{aligned}
& \mu_{A}=\varepsilon_{V}-R T \log \left(\frac{c_{S i}+\beta c_{L i}}{c_{A}-\left(c_{S i}+\beta c_{L i}\right)}\right)+\Omega_{V}\left\{\phi_{e}(\boldsymbol{\sigma})-\frac{1}{3} \operatorname{tr}(\boldsymbol{\sigma})\right\} \\
& \mu_{L i}=\sum_{n=1}^{N} \Lambda_{n} n\left(\frac{c_{L i}}{r c_{S i}}\right)^{n-1}+R T \log \left(\frac{c_{L i}}{r c_{S i}-c_{L i}}\right)-\beta \mu_{A}+\Omega_{L i}\left\{\phi_{e}(\boldsymbol{\sigma})-\frac{1}{3} \operatorname{tr}(\boldsymbol{\sigma})\right\} \\
& \mu_{S i}=\sum_{n=1}^{N} \Lambda_{n} r(1-n)\left(\frac{c_{L i}}{r c_{S i}}\right)^{n}-r R T \log \left(\frac{c_{L i}}{c_{L i}-r c_{S i}}\right)-\mu_{A}+\Omega_{S i}\left\{\phi_{e}(\boldsymbol{\sigma})-\frac{1}{3} \operatorname{tr}(\boldsymbol{\sigma})\right\}
\end{aligned}
$$

The vacancy formation energy $\varepsilon_{V}$ can be more conveniently be quantified by the equilibrium volume fraction of vacancies in stress free pure $\mathrm{Si} c_{V 0}$ through the relationship

$$
\varepsilon_{V}=R T \log \left(\frac{c_{S i 0}}{c_{V 0}}\right)
$$

The rate of vacancy nucleation can be expressed as 


$$
\frac{D n_{A}}{D t}=v_{0}^{p}\left(\frac{\sigma_{e}}{\sigma_{0}+v_{0}^{p} \mu_{A} / \dot{\varepsilon}_{0}}\right)^{m+1}-v_{0}^{T}\left(\frac{\left|\mu_{A}\right|}{R T}\right)^{n-1} \frac{\mu_{A}}{R T}
$$

The network velocity $\mathbf{v}$ can be eliminated from the conservation equations, with the result

$$
\begin{aligned}
& \frac{D c_{L i}}{D t}+c_{L i} \Omega_{V} \frac{D n_{A}}{D t}-\left(\Omega_{S i}-\Omega_{V}\right) c_{L i} \nabla \cdot \mathbf{j}_{S i}+\left[1-c_{L i}\left(\Omega_{L i}-\beta \Omega_{V}\right)\right] \nabla \cdot \mathbf{j}_{L i}=0 \\
& \frac{D c_{S i}}{D t}+c_{S i} \Omega_{V} \frac{D n_{A}}{D t}-\left(\Omega_{L i}-\beta \Omega_{V}\right) c_{S i} \nabla \cdot \mathbf{j}_{L i}+\left[1-c_{S i}\left(\Omega_{S i}-\Omega_{V}\right)\right] \nabla \cdot \mathbf{j}_{S i}=0 \\
& \frac{D c_{A}}{D t}-\left(\Omega_{L i}-\beta \Omega_{V}\right) c_{A} \nabla \cdot \mathbf{j}_{L i}-c_{A}\left(\Omega_{S i}-\Omega_{V}\right) \nabla \cdot \mathbf{j}_{S i}=\left(1-c_{A} \Omega_{V}\right) \frac{D n_{A}}{D t}
\end{aligned}
$$

Finally, the mechanical stress-strain rate equations can be combined to yield

$$
\begin{aligned}
\left(\nabla \mathbf{v}+\nabla \mathbf{v}^{T}\right) / 2 & =\frac{1}{2 \mu}\left(\underset{\boldsymbol{\sigma}}{-}-\frac{v}{1+v} \operatorname{tr}(\boldsymbol{\sigma}) \mathbf{I}\right)+\dot{\varepsilon}_{0}\left(\frac{\sigma_{e}}{\sigma_{f}\left(\mu_{A}\right)}\right)^{m} \frac{3}{2} \frac{\mathbf{S}}{\sigma_{e}} \\
& -\left(\left(\Omega_{L i}-\beta \Omega_{V}\right) \nabla \cdot \mathbf{j}_{L i}+\left(\Omega_{S i}-\Omega_{V}\right) \nabla \cdot \mathbf{j}_{S i}-\Omega_{V} \frac{D n_{A}}{D t}\right) \frac{\mathbf{I}}{3}
\end{aligned}
$$

Equations (33)-(37), together with the transport equations (30), (31); the conservation equations (3), (4), and the mechanical equilibrium equation $\nabla \cdot \boldsymbol{\sigma}+m \mathbf{b}=\mathbf{0}$ then yield a set of field equations for the concentrations $\left\{c_{L i}, c_{S i}, c_{A}\right\}$; the lattice velocity $\mathbf{v}$ and fluxes $\mathbf{j}_{L i}, \mathbf{j}_{S i}$ and stress $\boldsymbol{\sigma}$. The equations are approximate, and will be accurate only if the pressure is small compared with the bulk modulus $\mu(1+v) /(1-2 v)>>|\operatorname{tr}(\boldsymbol{\sigma})|$. This is likely to be the case in most practical electrode microstructures.

\section{Discussion}

We next explore the predictions of the model described in the preceding section. We will not attempt to solve (33)-(37) in detail, but will focus on simple model problems that illustrate the roles played by some of the parameters and constitutive equations that do not appear in classical models of diffusion in solids.

To enable comparisons with experiment, we consider cyclic lithiation of a thin-film electrode that is bonded to a rigid substrate. The film has thickness $h=h_{0}$ at time $t=0 . \mathrm{Li}$ is inserted into the film with a molar flux of Li per unit surface area $Q_{L i}(t)$ inserted into the film. As a specific example, we consider a charge-discharge cycle

To make the calculations more specific, we estimate values for as many material parameters in the model as possible from previously published work. Representative values are listed in Table 1.

For simplicity we assume that the Li insertion rate and film thickness are sufficiently small to ensure a uniform composition throughout the film. The film is then in a state of biaxial stress, $\sigma_{11}=\sigma_{22}=\sigma \quad \sigma_{33}=0$; the hydrostatic and deviatoric stresses $\operatorname{are} \operatorname{tr}(\boldsymbol{\sigma})=2 \sigma$ and $\sigma_{e}=\sigma$. Averaging (36) over the film thickness yields 


$$
\begin{aligned}
& \frac{d c_{L i}}{d t}=\left[1-c_{L i}\left(\Omega_{L i}-\beta \Omega_{V}\right)\right] \frac{Q_{L i}(t)}{h}-c_{L i} \Omega_{V} \frac{d n_{A}}{d t} \\
& \frac{d c_{S i}}{d t}=-\left(\Omega_{L i}-\beta \Omega_{V}\right) c_{S i} \frac{Q_{L i}(t)}{h}-c_{S i} \Omega_{V} \frac{d n_{A}}{d t} \\
& \frac{d c_{A}}{d t}=\left(1-c_{A} \Omega_{V}\right) \frac{d n_{A}}{d t}-\left(\Omega_{L i}-\beta \Omega_{V}\right) c_{A} \frac{Q_{L i}(t)}{h}
\end{aligned}
$$

while the in-plane and out of plane components of (37) yield equations for the evolution of stress and film thickness

$$
\begin{aligned}
& \frac{1-v}{2 \mu(1+v)} \frac{d \sigma}{d t}=-\dot{\varepsilon}_{0}\left(\frac{|\sigma|}{\sigma_{f}\left(\mu_{A}\right)}\right)^{m-1} \frac{\sigma}{\sigma_{f}\left(\mu_{A}\right)}-\frac{1}{3}\left(\left(\Omega_{L i}-\beta \Omega_{V}\right) \frac{Q_{L i}(t)}{h}+\Omega_{V} \frac{d n_{A}}{d t}\right) \\
& \frac{1}{h} \frac{d h}{d t}=\left(\left(\Omega_{L i}-\beta \Omega_{V}\right) \frac{Q_{L i}(t)}{h}+\Omega_{V} \frac{d n_{A}}{d t}\right)
\end{aligned}
$$

The small elastic thickness change has been neglected. Eqs. (38) and (39), together with (33) and (35) provide a system of equations that predict the evolution of stress, film thickness and concentration given a history of Li flux. As a specific example, we consider a half-cell, consisting of a 100nm a-Si film, with properties listed in Table 1, and an Li counter-electrode. The film is repeatedly lithiated galvanostatically at a current density of $8.1 \mu \mathrm{A} / \mathrm{cm}^{-2}$ using the cycle shown in Fig. 2. We estimate the potential of the thin film electrode relative to the $\mathrm{Li}$ counter-electrode by assuming local equilibrium at both electrode/electrolyte interfaces and neglecting losses in the electrolyte, in which case

$$
U \approx-\sum_{n=1}^{N} \frac{\Lambda_{n}}{F} n\left(\frac{c_{L i}}{r c_{S i}}\right)^{n-1}-\frac{R T}{F} \log \left(\frac{c_{L i}}{r c_{S i}-c_{L i}}\right)+\frac{\beta}{F} \mu_{A}+\frac{2 \Omega_{L i} \sigma}{3 F}-\frac{(1-v) \Omega_{L i} \sigma^{2}}{2 \mu F}
$$

where $F$ is the Faraday constant.

It is helpful to begin with a brief review of the predictions of prior models of amorphous thin-film $\mathrm{Si}$ electrodes in a half-cell, based on classical models of diffusion in solids. With an appropriate choice of material parameters, the extended model here reduces to the classical form. For example, setting $\beta=0$ (which makes Li fully interstitial); $\dot{\varepsilon}_{0}=0$ (which makes the material behavior elastic) and $v_{0}^{T}=0$ (which keeps the number of Si sites fixed) reduces the model to the elastic Larche-Cahn model. Selecting a finite value for $\dot{\varepsilon}_{0}$, but keeping $\beta=v_{0}^{p}=v_{0}^{T}=0$ produces the elastic-plastic models of Li-Si used in refs $[14,15,18]$, among others. For reference, the predicted voltage, stress, concentration and film thickness in an an amorphous Si film idealized with this model are shown in Fig. 2. Li inserted into the film causes it to expand. The expansion of the film in its own plane is resisted by the substrate, which develops a large stress in the film (Fig 2b). When the stress reaches yield, the film begins to deform plastically. The stress-v-time curve ceases to be linear at this point. The plastic flow causes a permanent contraction of the film in its own plane. Conventional treatments of plasticity assume that this plastic flow does not change the volume of the film, so the in-plane contraction is balanced by a corresponding increase in thickness of the film (Fig 2d). After yield, all the additional Li is accommodated by a permanent increase in the film thickness. When the $\mathrm{Li}$ is removed from the film, this process is reversed. The film contracts; this induces tensile stresses, which eventually become large enough to cause the film to yield in tension. Further Li removal causes the film to be stretched permanently in its own plane, and the film thickness decreases so as to conserve volume. The film returns to its original thickness when fully de-lithiated. 
With this preamble, we now examine the consequences of the changes in Si structure, and illustrate the effects of the material parameters $c_{V 0}$ (or equivalently $\varepsilon_{V}$ ), $\beta$, and the vacancy nucleation parameters $v_{0}^{p}=v_{0}^{T}$. The latter are conveniently parameterized by two dimensionless nucleation rates

$$
\mathrm{N}_{p}=\left(\frac{Q_{L i}}{c_{S i 0} h_{0} \dot{\varepsilon}_{0}}\right)^{1 / m} \frac{v_{0}^{p}}{c_{S i 0} \dot{\varepsilon}_{0}} \quad \mathrm{~N}_{T}=\frac{h_{0} v_{0}^{T}}{Q_{L i}}
$$

Consider first the case $\mathrm{N}_{p}=0, \beta=0$. This implies that plastic flow does not create unoccupied Si sites, and all inserted $\mathrm{Li}$ is accommodated at interstitial sites. In this case, if the solid were to remain stress free, it would expand reversibly, the number of vacant Si sites remains constant, and energy would be dissipated only through diffusion (and possibly the Li insertion reaction at the surface, which is not considered here). A thin film electrode prevented from expanding in the plane of the film by the substrate, and consequently develops compressive stress when $\mathrm{Li}$ is inserted. In $\mathrm{Li}-\mathrm{Si}$ the stresses are usually sufficient to cause irreversible plastic flow in the film. The compressive stress tends to result in vacant $\mathrm{Si}$ sites being absorbed. When $\mathrm{Li}$ is subsequently removed from the film, the stresses become tensile, and cause Si sites to be nucleated. The predicted variations of potential, stress, concentration of vacant Si sites, and film thickness are shown in Fig. 3, for two values of the equilibrium vacant site concentration $c_{V 0}$, and for $\mathrm{N}_{T}=0.1$, which corresponds to an intermediate rate of vacancy nucleation in comparison to the $\mathrm{Li}$ insertion rate. Nucleation of vacant sites results in some minor additional features on the variations of stress and potential. The most striking effect, however, is that the Li film thickness permanently increases after the first lithiation cycle. The change in film thickness in this limit is a consequence of stress driven vacancy nucleation: a small value of $c_{V 0}$ implies a high energetic cost to nucleating vacant sites, and consequently results in only a small thickness change. For large values of $c_{V 0}$ significant change in thickness may occur.

Consider next the limit $\mathrm{N}_{p}=0, \beta=0.7$. With this choice all the inserted Li must be accommodated by creating additional vacant sites in the $\mathrm{Si}$. Creating vacant $\mathrm{Si}$ sites dissipates energy, so $\mathrm{Li}$ insertion is irreversible even in a stress free solid. Behavior in this limit is similar to the predictions of the 'reaction controlled' diffusion model proposed by Brassart and Suo [29], in which the usual relationship between Li chemical potential and free energy in eq (33) is replaced by an equation relating the time derivative of concentration to a generalized chemical potential. Our idealization here uses the conventional expressions for all thermodynamic driving forces, and attributes the irreversible reaction to the rearrangement of the Si network rather than the Li insertion process itself. Nevertheless, it yields a very similar relationship between $\mathrm{Li}$ chemical potential and concentration. For example, for the particular case $n=1$ and $v_{0}^{p}=0$ in eq. (35), and assuming Si is immobile, and assuming the solid is stress free, eqs (33), (35) and (36) can be combined to yield

$$
\mu_{L i}=\sum_{n=1}^{N} \Lambda_{n} n\left(\frac{c_{L i}}{r c_{S i}}\right)^{n-1}+R T \log \left(\frac{c_{L i}}{r c_{S i}-c_{L i}}\right)-\frac{\beta R T}{c_{L i} \Omega_{V} v_{0}^{T}}\left(\frac{D c_{L i}}{D t}+\left[1-c_{L i}\left(\Omega_{L i}-\beta \Omega_{V}\right)\right] \nabla \cdot \mathbf{j}_{L i}\right)
$$

showing that, away from equilibrium, the Li chemical potential depends on both concentration and its time derivative.

The energy losses resulting from the structural changes accompanying changes in Li concentration can be visualized by plotting the irreversible potential-v-capacity curves: the area enclosed by the hysteresis loss is the lost energy. Representative results are shown in Fig 3 for both a stress free material, with $n=1$ and $n=10$ in eq. (35), as well as for a thin film electrode. These results do not include losses resulting from the electrochemical reaction. In a stress free material with $n=1$ the voltage-v-capacity loss is nearly reversible, suggesting that structural changes are not a significant source of energy loss in this limit, but 
for values of $n>1$ in can become significant. In a thin film electrode, the material deforms plastically in compression during Li insertion, and in tension during removal (see, e.g. Fig 2b). Fig 4 suggests that losses resulting from plastic flow greatly exceed those associated with irreversible volume changes.

We next investigate the effects of vacant $\mathrm{Si}$ sites produced by stress-driven plastic flow. With $\mathrm{N}_{P}>0$, stress driven plastic flow generates free volume, and results in both an irreversible shear strain, together with an increase in volume. The change in volume is permanent if the Si lattice structure is assumed to be unaltered by thermal activation $\left(\mathrm{N}_{T}=0\right)$. With $\mathrm{N}_{T}>0$, the $\mathrm{Si}$ lattice can recover towards its equilibrium vacancy concentration by thermal activation. In addition, if $\mathrm{N}_{P}>0$ the flow stress of Si depends on the Si vacancy chemical potential through the second of eq. (28). The most significant consequences of plastic flow induced vacancy nucleation are therefore on the variation of stress, vacancy concentration, and film thickness, which are illustrated in Fig 5, for two values of $\mathrm{N}_{T}$. If plasticity induced vacancy nucleation greatly exceeds the rate of thermally activated vacancy nucleation, the vacancy concentration progressively increases with time, and causes a gradual increase in film thickness with each lithiation cycle. The resulting decrease in vacancy chemical potential also causes a significant difference between the tensile and compressive flow stress in the film, and also results in a gradual increase in both tensile and compressive flow stress with cycling. Similar tension-compression asymmetry has been predicted by molecular dynamic simulations [25]. With a nonzero value for $\mathrm{N}_{T}$, the nucleation of vacant sites is (after a transient) balanced by thermally activated recovery. In this case the film increases its thickness during the first cycle, and thereafter cycles between fixed limits. The compressive flow stress remains greater than the tensile flow stress, but the asymmetry is reduced.

Several of these predictions are in good qualitative agreement with experimental measurements. Tokranov et al [27] have reported a $25 \%$ increase in the thickness of an Si film after the first cycle of lithiation, in agreement with the predictions shown in Fig. 5(d). Thickness measurements are complicated by the formation of SEI on the electrode surface, however, and further careful comparisons of measured and predicted shape changes resulting from Li insertion would be valuable. Several experiments have observed differences between the flow stress of Li-Si during insertion and de-lithiation, but these observations have previously been attributed to the effects of initial deposition stresses in the $\mathrm{Si}$ films $[13,14]$. Further more detailed comparisons between theory and experiment will be necessary to determine which explanation is correct.

We conclude by investigating the predicted variation of flow stress with $\mathrm{Li}$ insertion rate. This is of interest because the rate sensitivity provides some insight into the $\mathrm{Li}$ insertion process. For example, reactive flow models [29] predict the flow stress should reduce with insertion rate, while models based on classical plasticity theory predict the opposite $[14,15]$. Two different approaches have been used to estimate the strain rate sensitivity of Si experimentally. Bucci et al subjected a thin film electrode to successive Li insertion cycles at progressively increasing rate. Pharr et al used jump tests, in which the Li insertion rate was varied in a succession of steps during a single insertion cycle. The predicted variations of stress in both types of experiment are illustrated in Fig. 6. The rate sensitivity of flow can be estimated by plotting the variation of flow stress with $\mathrm{Li}$ insertion rate, as shown in Fig. 7. The flow stress is predicted to increase with charging rate, in agreement with experiment. The variation of both tensile and compressive flow stress in the cyclic tests can be fit by a power-law dependence $\sigma=C \dot{Q}^{a}$, with an exponent $a \approx 1 / 20$. The jump tests are more sensitive to transients associated with changes in the Si structure. The flow stresses resulting from step decreases in charging rate are more strongly rate sensitive than those resulting from step increases. An expression of the form $\sigma=\sigma_{1}+C \dot{Q}^{b}$ with $b \approx 1 / 3$ predicts behavior between the two limits. This may partly explain why the two experiments in refs. [14, 15] yielded different values for the stress exponent. 


\section{Conclusions}

We have described a generalized model of $\mathrm{Li}$ insertion and transport in amorphous $\mathrm{Si}$, which accounts for irreversible changes in the underlying atomic structure of the Si network during lithiation. We assume two mechanisms play a role in this process: first, free volume (quantified here by a concentration of unoccupied sites in the amorphous Si network) may be nucleated by a thermally activated process, driven by stress and solution chemistry. Secondly, plastic flow caused by sufficiently large stresses may also nucleate unoccupied Si sites.

As a representative example, the model was used to predict the evolution of potential, stress, composition and thickness of a 100nm thick amorphous Si electrode in a half-cell, subjected to several successive charge-discharge cycles. Losses resulting from transport in the film, and the electrochemical Li insertion reaction were neglected to simplify calculations.

The generalized model predicts several phenomena that are not captured by classical models of diffusion in solids. These include: (i) Li-Si may experience irreversible changes in volume after lithiation, as a result of changes in the Si network structure; (ii) the flow stress of $\mathrm{Li}-\mathrm{Si}$ in compression exceeds the flow stress in tension; (iii) energy may be dissipated as a result of irreversible changes in Si network structure even in a stress-free solid.

Several of these predictions are at least in qualitative agreement with recent experiments. For example, ref [27] has reported a permanent $25 \%$ increase in thickness of Si films after the first lithium insertion cycle, in good agreement with predictions shown in Fig. 4(d). Tension-compression asymmetry has been observed in several experiments [13, 14], but was previously attributed to the effects of stresses induced in the Si films by the deposition process.

Our results suggest several possible questions for future study. Firstly, our predictions are based on a set of speculative equations that quantify the changes in free energy in Li-Si resulting from changes in the Si structure, which may require revision. Secondly, the calculations reported here have not addressed Li transport through $\mathrm{Si}$ in detail, and in particular, have not considered how the diffusion coefficient may be altered by changes in the Si structure. Thirdly, the approach used here to quantify structural changes in Li-Si could be extended to consider the crystalline-amorphous transformation that occurs in crystalline $\mathrm{Si}$, possibly by making the free energy contribution from vacancies $g_{V}$ in eq. (22) a non-convex function of unoccupied site concentration.

The approach adopted here to model Li-Si may also have more general applications to describing structural changes in electrode materials. In particular, phase-field models of materials which experience phase transformations usually assume that variation of free energy of a solid with its composition can be expressed as a function of the Li concentration and its gradient. In this approach, the Li concentration characterizes both the composition of the material as well as its structure. In general, however, composition and structure can vary independently, and free energy of a solid is not a unique function of composition. The approach used here to quantify structural changes in amorphous Li-Si could be extended to consider more complex changes in crystal structure. In a general model of this form, the free energy of the solid would be a function of composition, one or more order parameters characterizing crystal structure, and the gradients of these order parameters. 


\section{Acknowledgements}

AFB, EC and PG were supported by the U.S. Department of Energy through DOE EPSCoR Implementation Grant no. DE-SC0007074. BWS was supported by General Motors through the Brown/GM Collaborative Research Laboratory for Computational Materials Research. AFB is grateful to Daniel Baker and Mark Verbrugge of General Motors R\&D Center for helpful discussions. 


\section{References}

[1] Zhang W-J. A review of the electrochemical performance of alloy anodes for lithium-ion batteries. Journal of Power Sources. 2011;196:13-24.

[2] Chan CK, Peng H, Liu G, Mcllwrath K, Zhang XF, Huggins RA, et al. High-performance lithium battery anodes using silicon nanowires. Nat Nano. 2008;3:31-5.

[3] Peng K, Jie J, Zhang W, Lee S-T. Silicon nanowires for rechargeable lithium-ion battery anodes. Applied Physics Letters. 2008;93:-.

[4] Haftbaradaran H, Xiao X, Verbrugge MW, Gao H. Method to deduce the critical size for interfacial delamination of patterned electrode structures and application to lithiation of thin-film silicon islands. Journal of Power Sources. 2012;206:357-66.

[5] Soni SK, Sheldon BW, Xiao X, Verbrugge MW, Dongjoon A, H. H, et al. Stress Mitigation during the Lithiation of Patterned Amorphous Si Islands. Journal of The Electrochemical Society. 2011;159:A38-A43.

[6] Wu H, Zheng G, Liu N, Carney TJ, Yang Y, Cui Y. Engineering Empty Space between Si Nanoparticles for Lithium-Ion Battery Anodes. Nano Letters. 2012;12:904-9.

[7] Zhao J, Lu Z, Liu N, Lee H-W, McDowell MT, Cui Y. Dry-air-stable lithium silicide-lithium oxide core-shell nanoparticles as high-capacity prelithiation reagents. Nat Commun. 2014;5.

[8] Mukhopadhyay A, Sheldon BW. Deformation and stress in electrode materials for Li-ion batteries. Progress in Materials Science. 2014;63:58-116.

[9] Obrovac MN, Krause LJ. Reversible Cycling of Crystalline Silicon Powder. Journal of The Electrochemical Society. 2007;154:A103-A8.

[10] Limthongkul P, Jang Y-I, Dudney NJ, Chiang Y-M. Electrochemically-driven solid-state amorphization in lithium-silicon alloys and implications for lithium storage. Acta Materialia. 2003;51:1103-13.

[11] McDowell MT, Ryu I, Lee SW, Wang C, Nix WD, Cui Y. Studying the Kinetics of Crystalline Silicon Nanoparticle Lithiation with In Situ Transmission Electron Microscopy. Advanced Materials. 2012;24:6034-41.

[12] Yang H, Huang S, Huang X, Fan F, Liang W, Liu XH, et al. Orientation-Dependent Interfacial Mobility Governs the Anisotropic Swelling in Lithiated Silicon Nanowires. Nano Letters. 2012;12:1953-8.

[13] Sethuraman VA, Chon MJ, Shimshak M, Srinivasan V, Guduru PR. In situ measurements of stress evolution in silicon thin films during electrochemical lithiation and delithiation. Journal of Power Sources. 2010;195:5062-6.

[14] Bucci G, Nadimpalli SPV, Sethuraman VA, Bower AF, Guduru PR. Measurement and modeling of the mechanical and electrochemical response of amorphous Si thin film electrodes during cyclic lithiation. Journal of the Mechanics and Physics of Solids. 2014;62:276-94.

[15] Pharr M, Suo Z, Vlassak JJ. Variation of stress with charging rate due to strain-rate sensitivity of silicon electrodes of Li-ion batteries. Journal of Power Sources. 2014;270:569-75.

[16] Berla LA, Lee SW, Cui Y, Nix WD. Mechanical behavior of electrochemically lithiated silicon. Journal of Power Sources. 2015;273:41-51.

[17] Cui Z, Gao F, Qu J. A finite deformation stress-dependent chemical potential and its applications to lithium ion batteries. Journal of the Mechanics and Physics of Solids. 2012;60:1280-95.

[18] Bower AF, Guduru PR, Sethuraman VA. A finite strain model of stress, diffusion, plastic flow, and electrochemical reactions in a lithium-ion half-cell. Journal of the Mechanics and Physics of Solids. 2011;59:804-28.

[19] Anand L. A Cahn-Hilliard-type theory for species diffusion coupled with large elastic-plastic deformations. Journal of the Mechanics and Physics of Solids. 2012;60:1983-2002.

[20] Yang H, Fan F, Liang W, Guo X, Zhu T, Zhang S. A chemo-mechanical model of lithiation in silicon. Journal of the Mechanics and Physics of Solids. 2014;70:349-61. 
[21] Brassart L, Zhao K, Suo Z. Cyclic plasticity and shakedown in high-capacity electrodes of lithium-ion batteries. International Journal of Solids and Structures. 2013;50:1120-9.

[22] Zhao K, Pharr M, Cai S, Vlassak JJ, Suo Z. Large Plastic Deformation in High-Capacity LithiumIon Batteries Caused by Charge and Discharge. Journal of the American Ceramic Society. 2011;94:s226-s35.

[23] Huang S, Fan F, Li J, Zhang S, Zhu T. Stress generation during lithiation of high-capacity electrode particles in lithium ion batteries. Acta Materialia. 2013;61:4354-64.

[24] Zhao K, Tritsaris GA, Pharr M, Wang WL, Okeke O, Suo Z, et al. Reactive Flow in Silicon Electrodes Assisted by the Insertion of Lithium. Nano Letters. 2012;12:4397-403.

[25] Feifei F, Shan H, Hui Y, Muralikrishna R, Dibakar D, Vivek BS, et al. Mechanical properties of amorphous Li x Si alloys: a reactive force field study. Modelling and Simulation in Materials Science and Engineering. 2013;21:074002.

[26] Wang JW, He Y, Fan F, Liu XH, Xia S, Liu Y, et al. Two-Phase Electrochemical Lithiation in Amorphous Silicon. Nano Letters. 2013;13:709-15.

[27] Tokranov A, Sheldon BW, Li C, Minne S, Xiao X. In Situ Atomic Force Microscopy Study of Initial Solid Electrolyte Interphase Formation on Silicon Electrodes for Li-Ion Batteries. ACS Applied Materials \& Interfaces. 2014;6:6672-86.

[28] BRASSART L, SUO Z. REACTIVE FLOW IN LARGE-DEFORMATION ELECTRODES OF LITHIUMION BATTERIES. International Journal of Applied Mechanics. 2012;04:1250023.

[29] Brassart L, Suo Z. Reactive flow in solids. Journal of the Mechanics and Physics of Solids. 2013;61:61-77.

[30] Zhang T, Guo Z, Wang Y, Zhu J. Effect of reversible electrochemical reaction on Li diffusion and stresses in cylindrical Li-ion battery electrodes. Journal of Applied Physics. 2014;115:-.

[31] Drozdov AD. Viscoplastic response of electrode particles in Li-ion batteries driven by insertion of lithium. International Journal of Solids and Structures. 2014;51:690-705.

[32] Larché F, Cahn JW. A linear theory of thermochemical equilibrium of solids under stress. Acta Metallurgica. 1973;21:1051-63.

[33] Larché F, Cahn JW. A nonlinear theory of thermochemical equilibrium of solids under stress. Acta Metallurgica. 1978;26:53-60.

[34] Larche FC, Cahn JW. Thermochemical equilibrium of multiphase solids under stress. Acta Metallurgica. 1978;26:1579-89.

[35] Greer AL, Spaepen F. CREEP, DIFFUSION, AND STRUCTURAL RELAXATION IN METALLIC GLASSES*. Annals of the New York Academy of Sciences. 1981;371:218-37.

[36] Spaepen F. A microscopic mechanism for steady state inhomogeneous flow in metallic glasses. Acta Metallurgica. 1977;25:407-15.

[37] Anand L, Su C. A constitutive theory for metallic glasses at high homologous temperatures. Acta Materialia. 2007;55:3735-47.

[38] Datta R, Vilekar SA. The continuum mechanical theory of multicomponent diffusion in fluid mixtures. Chemical Engineering Science. 2010;65:5976-89.

[39] $\mathrm{Wu} \mathrm{CH}$. The role of Eshelby stress in composition-generated and stress-assisted diffusion. Journal of the Mechanics and Physics of Solids. 2001;49:1771-94.

[40] Chevrier VL, Dahn JR. First Principles Model of Amorphous Silicon Lithiation. Journal of The Electrochemical Society. 2009;156:A454-A8.

[41] Bower AF. Applied Mechanics of Solids. Boca Raton, FL: CRC Press; 2010.

[42] John C, John N. Stress generation and fracture in lithium insertion materials. Journal of Solid State Electrochemistry. 2006;10:293-319.

[43] Gurtin MEF, Eliot; Anand, Lallit The Mechanics and Thermodynamics of Continua. Cambridge: Cambridge University Press; 2013.

[44] Truesdell C. Mechanical Basis of Diffusion. The Journal of Chemical Physics. 1962;37:2336-44. 
[45] Manning JR. Correlation Factors for Diffusion in Nondilute Alloys. Physical Review B. 1971;4:1111-21.

[46] Roorda S, Sinke WC, Poate JM, Jacobson DC, Dierker S, Dennis BS, et al. Structural relaxation and defect annihilation in pure amorphous silicon. Physical Review B. 1991;44:3702-25.

\section{List of Figure Captions}

Fig. 1: Schematic illustrating the idealized network model used to describe transport, microstructure evolution and deformation in amorphous Li-Si. The network consists of a set of Si sites, which may be occupied by $\mathrm{Si}$ atoms, by $\mathrm{Li}$ atoms, or may remain vacant. The Si sites may be created or annihilated. $\mathrm{Li}$ atoms may also occupy interstitial sites. The parameter $\beta$ in eq. (1) specifies the fraction of Li atoms that occupy Si sites.

Fig. 2: Predicted cycles of (a) potential; (b) stress (c) Unoccupied Si site concentration (normalized by the equilibrium concentration) and (d) film thickness (normalized by initial film thickness) for a thin film a-Si electrode in a half-cell with $\mathrm{Li}$ counter-electrode subjected to four successive charge-discharge cycles. Results are shown for $\beta=0, \mathrm{~N}_{p}=0, \mathrm{~N}_{T}=0$ and parameters listed in Table 1. In this limit no changes occur to the Si lattice structure.

Fig. 3: Predicted cycles of (a) potential; (b) stress (c) Unoccupied Si site concentration (normalized by the equilibrium concentration) and (d) film thickness (normalized by initial film thickness) for a thin film a-Si electrode in a half-cell with $\mathrm{Li}$ counter-electrode subjected to four successive charge-discharge cycles. Results are shown for $\beta=0, \mathrm{~N}_{p}=0, \mathrm{~N}_{T}=0.1$ and parameters listed in Table 1. Red solid line: $c_{V 0} / c_{S i 0}=0.01 ;$ blue dashed line $c_{V 0} / c_{S i 0}=0.001$.

Fig. 4: Predicted potential-v-capacity curves for a-Si subjected to a single cycle of Li insertion. Results are shown for $\beta=0.7, \mathrm{~N}_{p}=0, \mathrm{~N}_{T}=1.5$ and parameters listed in Table 1. (i) Results for a thin-film electrode with parameters listed in Table 1; (ii), (iii) Results for a stress-free solid with $n=1$ and $n=10$, respectively.

Fig. 5: Predicted cycles of (a) potential; (b) stress (c) Unoccupied Si site concentration (normalized by the equilibrium concentration) and (d) film thickness (normalized by initial film thickness) for a thin film a-Si electrode in a half-cell with $\mathrm{Li}$ counter-electrode subjected to four successive charge-discharge cycles. Results are shown for $\beta=0, c_{V 0} / c_{S i 0}=0.003$, and parameters listed in Table 1. Red solid line: $\mathrm{N}_{p}=0.2 \mathrm{~N}_{T}=0$; blue dashed line $\mathrm{N}_{p}=0.6 \mathrm{~N}_{T}=0.1$

Fig. 6: Predicted variations of stress resulting from (a) cyclic lithiation at increasing current density; and (b) step changes in lithiation current. Results are shown for $\beta=0, c_{V 0} / c_{S i 0}=0.003, \mathrm{~N}_{p}=0.8$, $\mathrm{N}_{p}=0.3$ and parameters listed in Table 1.

Fig. 7: Predicted variation of flow stress magnitude with $\mathrm{Li}$ insertion current, re-plotted from Fig 6. 


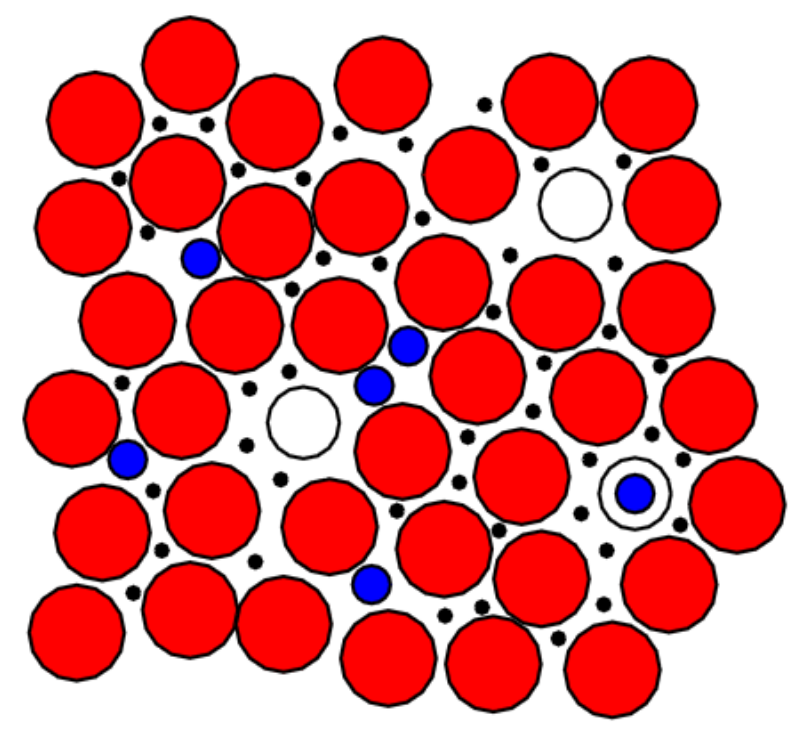

Si atom

Vacant Si

Site

Li atom

Vacant Li

Site

Li Occupying

Vacant Si Site
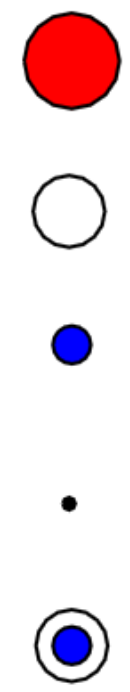

Fig. 1: Schematic illustrating the idealized network model used to describe transport, microstructure evolution and deformation in amorphous Li-Si. The network consists of a set of Si sites, which may be occupied by $\mathrm{Si}$ atoms, by $\mathrm{Li}$ atoms, or may remain vacant. The $\mathrm{Si}$ sites may be created or annihilated. $\mathrm{Li}$ atoms may also occupy interstitial sites. The parameter $\beta$ in eq. (1) specifies the fraction of $\mathrm{Li}$ atoms that occupy Si sites. 


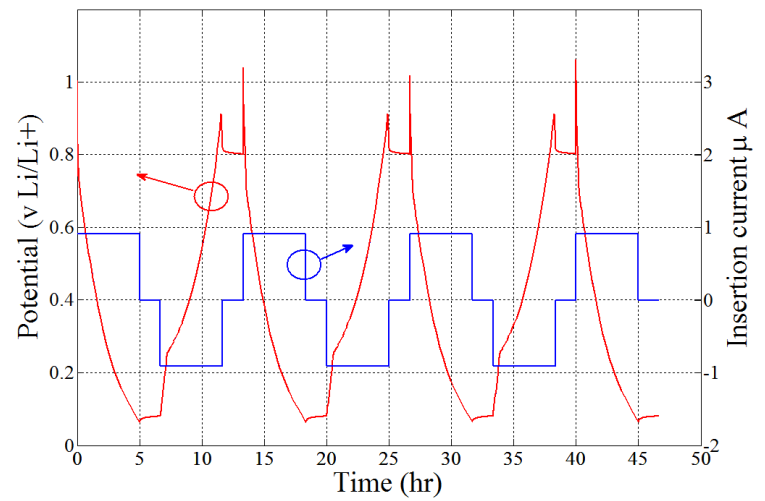

(a)

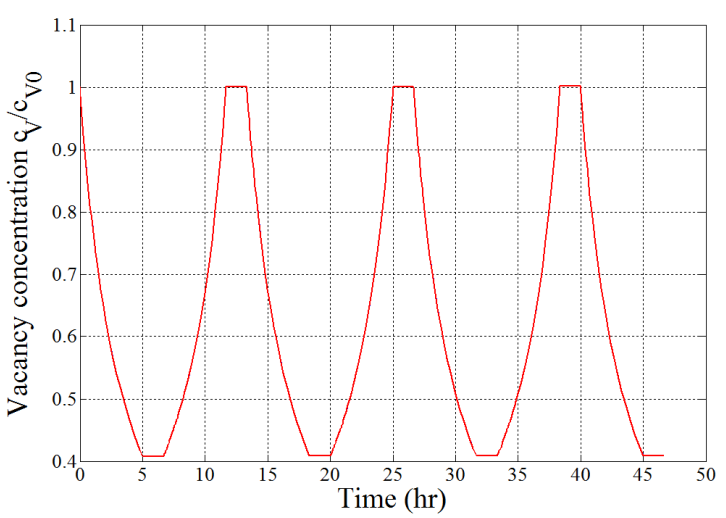

(c)

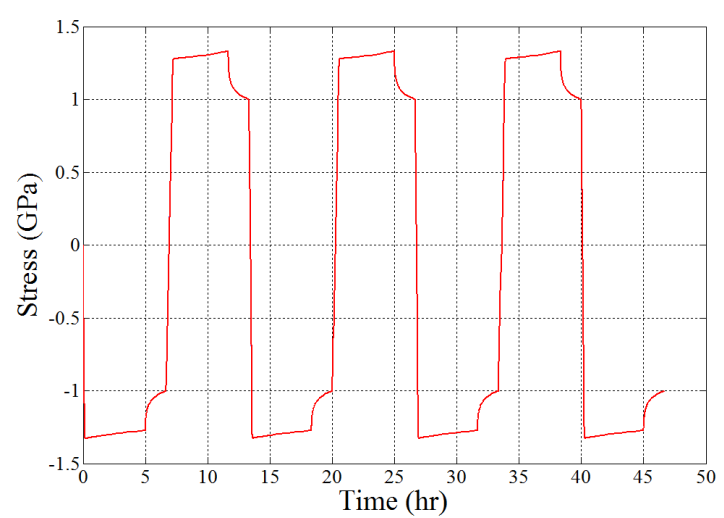

(b)

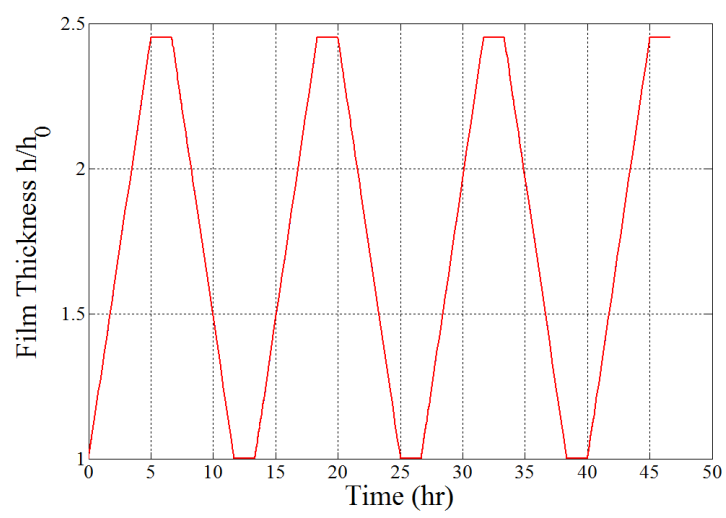

(d)

Fig. 2: Predicted cycles of (a) potential; (b) stress (c) Unoccupied Si site concentration (normalized by the equilibrium concentration) and (d) film thickness (normalized by initial film thickness) for a thin film a-Si electrode in a half-cell with $\mathrm{Li}$ counter-electrode subjected to four successive charge-discharge cycles. Results are shown for $\beta=0, \mathrm{~N}_{p}=0, \mathrm{~N}_{T}=0$ and parameters listed in Table 1. In this limit no changes occur to the Si lattice structure. 


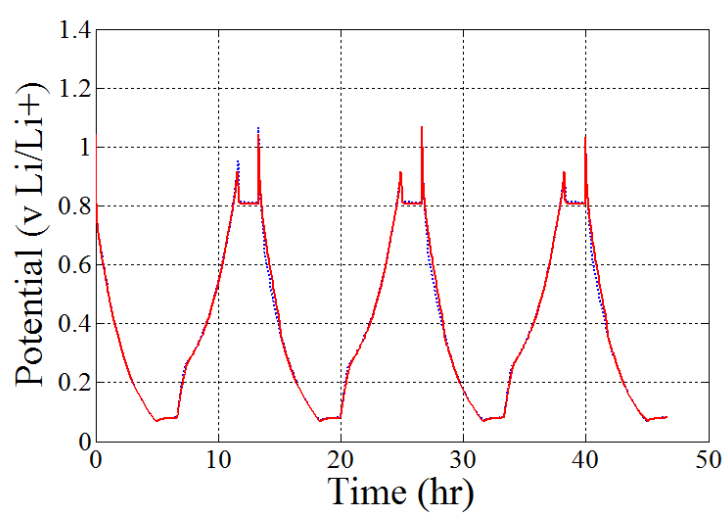

(a)

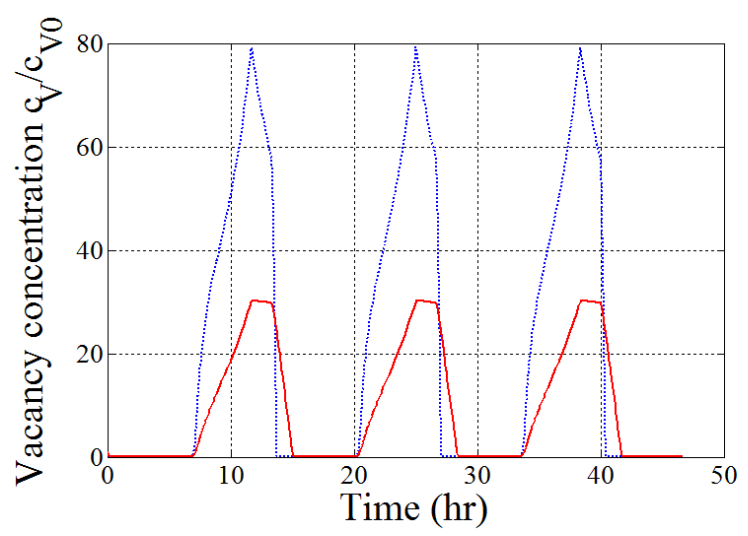

(c)

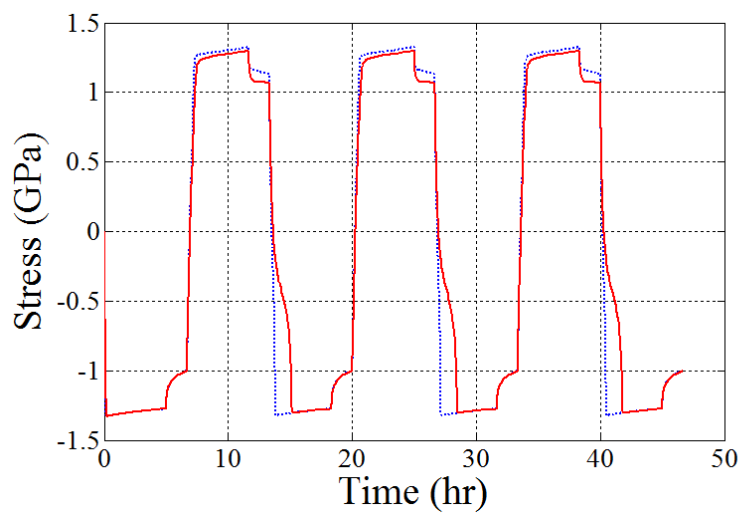

(b)

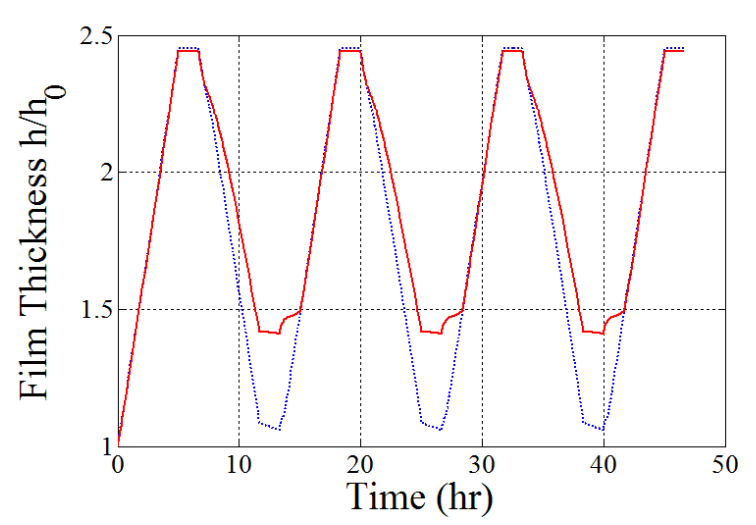

(d)

Fig. 3: Predicted cycles of (a) potential; (b) stress (c) Unoccupied Si site concentration (normalized by the equilibrium concentration) and (d) film thickness (normalized by initial film thickness) for a thin film a-Si electrode in a half-cell with Li counter-electrode subjected to four successive charge-discharge cycles. Results are shown for $\beta=0, \mathrm{~N}_{p}=0, \mathrm{~N}_{T}=0.1$ and parameters listed in Table 1. Red solid line: $c_{V 0} / c_{S i 0}=0.01 ;$ blue dashed line $c_{V 0} / c_{S i 0}=0.001$ 


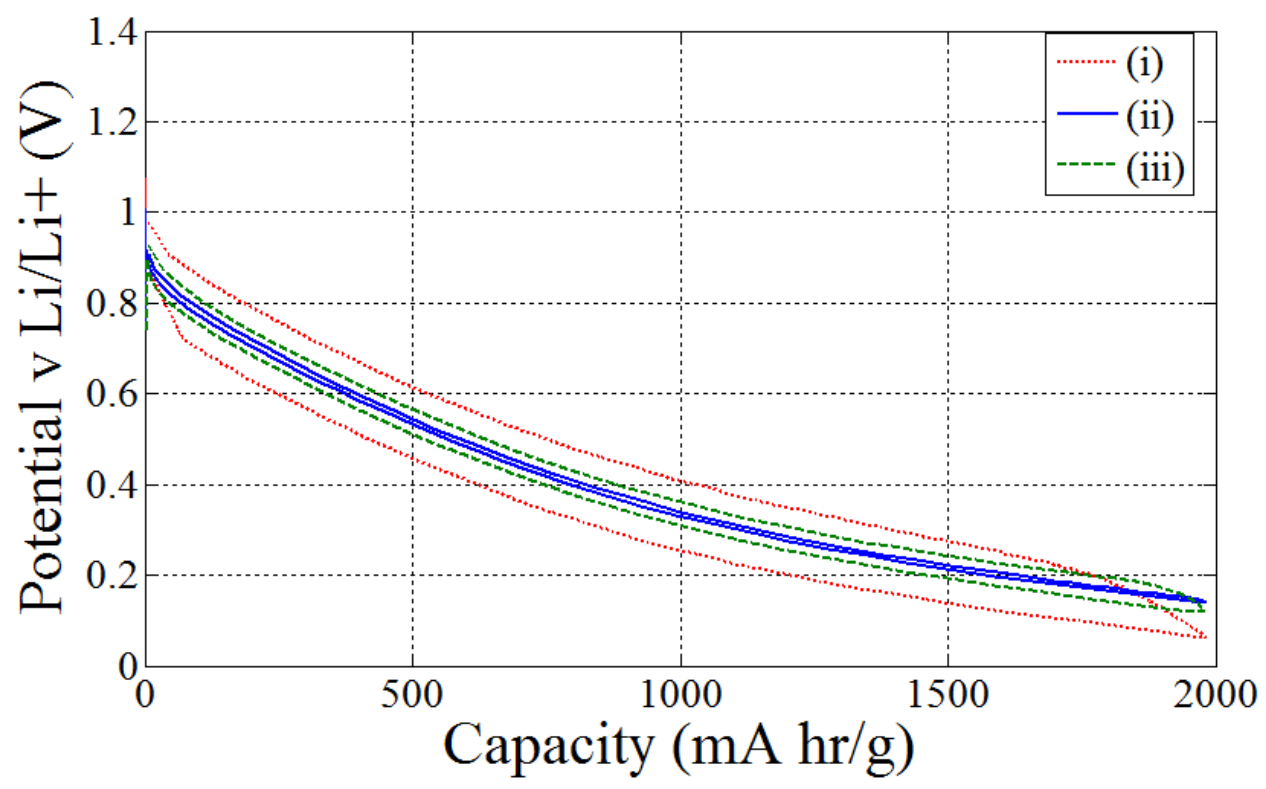

Fig. 4: Predicted potential-v-capacity curves for a-Si subjected to a single cycle of Li insertion. Results are shown for $\beta=0.7, \mathrm{~N}_{p}=0, \mathrm{~N}_{T}=1.5$ and parameters listed in Table 1. (i) Results for a thin-film electrode with parameters listed in Table 1; (ii), (iii) Results for a stress-free solid with $n=1$ and $n=10$, respectively. 


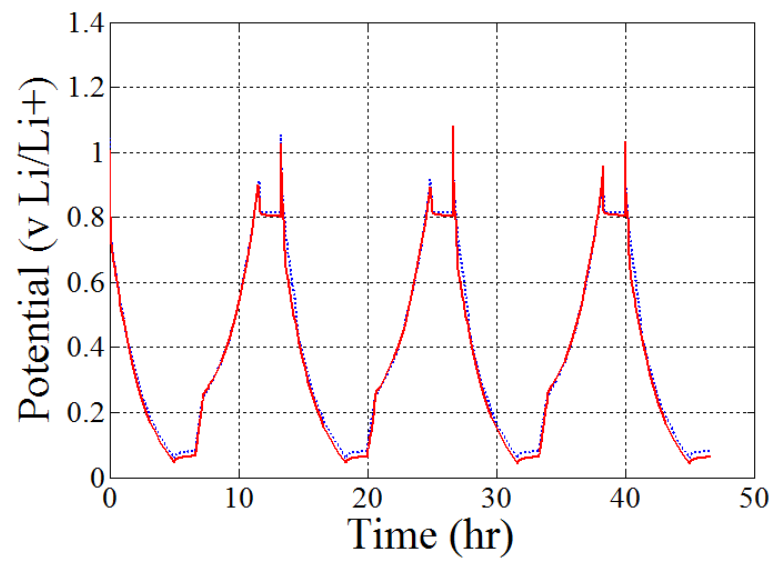

(a)

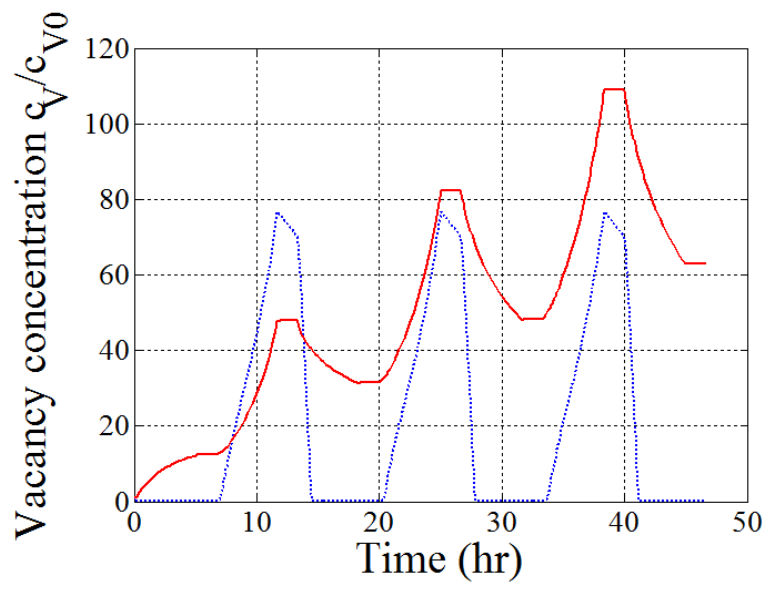

(c)

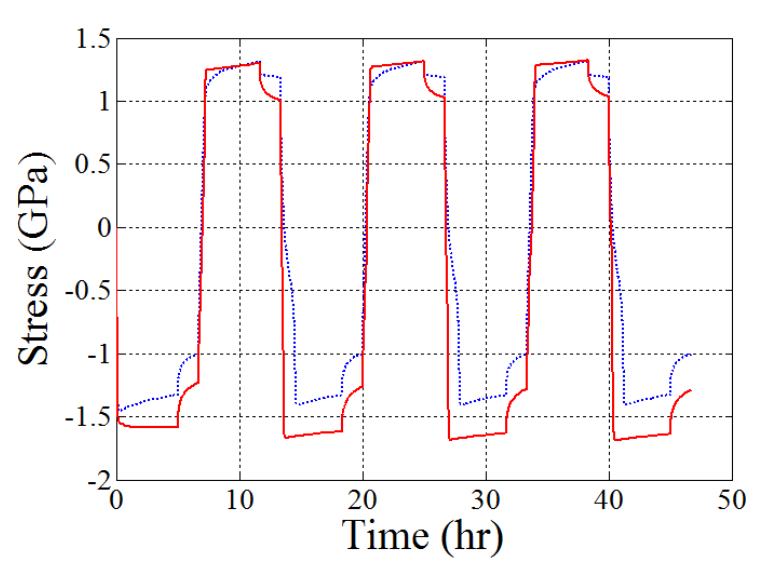

(b)

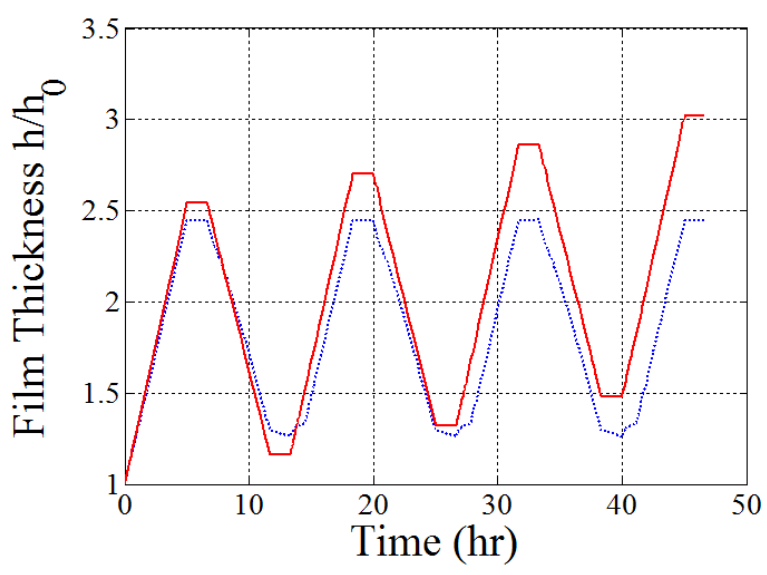

(d)

Fig. 5: Predicted cycles of (a) potential; (b) stress (c) Unoccupied Si site concentration (normalized by the equilibrium concentration) and (d) film thickness (normalized by initial film thickness) for a thin film a-Si electrode in a half-cell with $\mathrm{Li}$ counter-electrode subjected to four successive charge-discharge cycles. Results are shown for $\beta=0, c_{V 0} / c_{S i 0}=0.003$, and parameters listed in Table 1. Red solid line: $\mathrm{N}_{p}=0.2 \mathrm{~N}_{T}=0$; blue dashed line $\mathrm{N}_{p}=0.6 \mathrm{~N}_{T}=0.1$ 

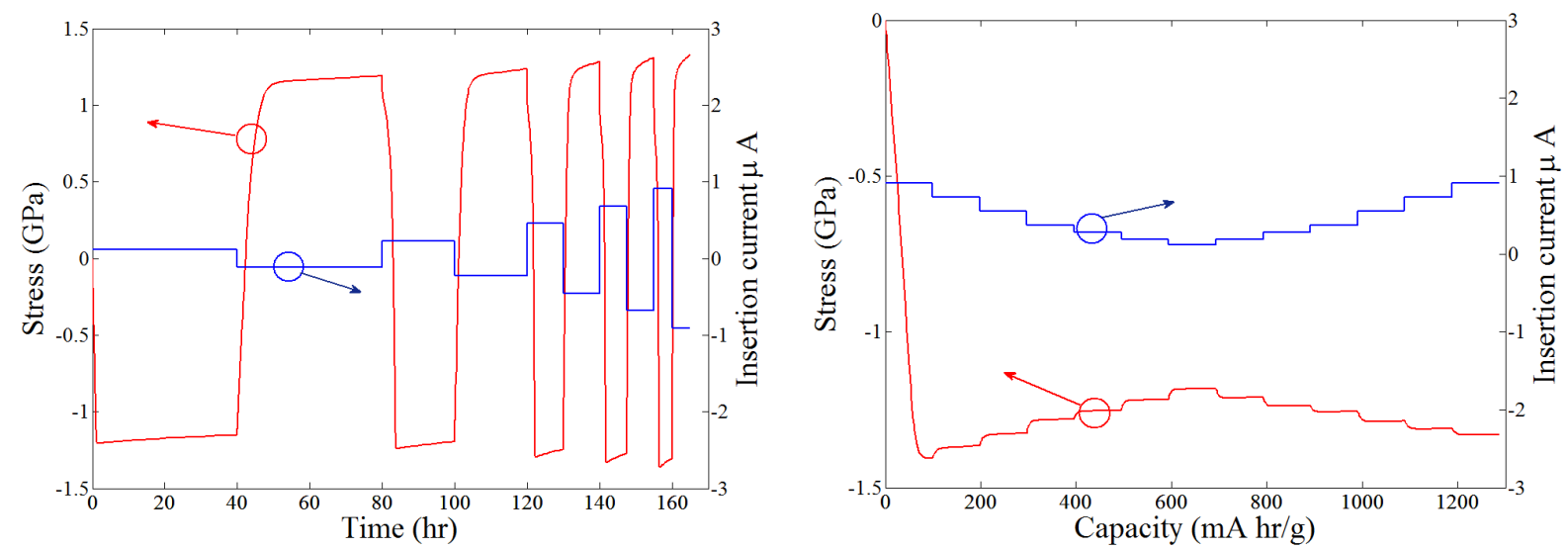

Fig. 6: Predicted variations of stress resulting from (a) cyclic lithiation at increasing current density; and (b) step changes in lithiation current. Results are shown for $\beta=0, c_{V 0} / c_{S i 0}=0.003, \mathrm{~N}_{p}=0.8$, $\mathrm{N}_{p}=0.3$ and parameters listed in Table 1.

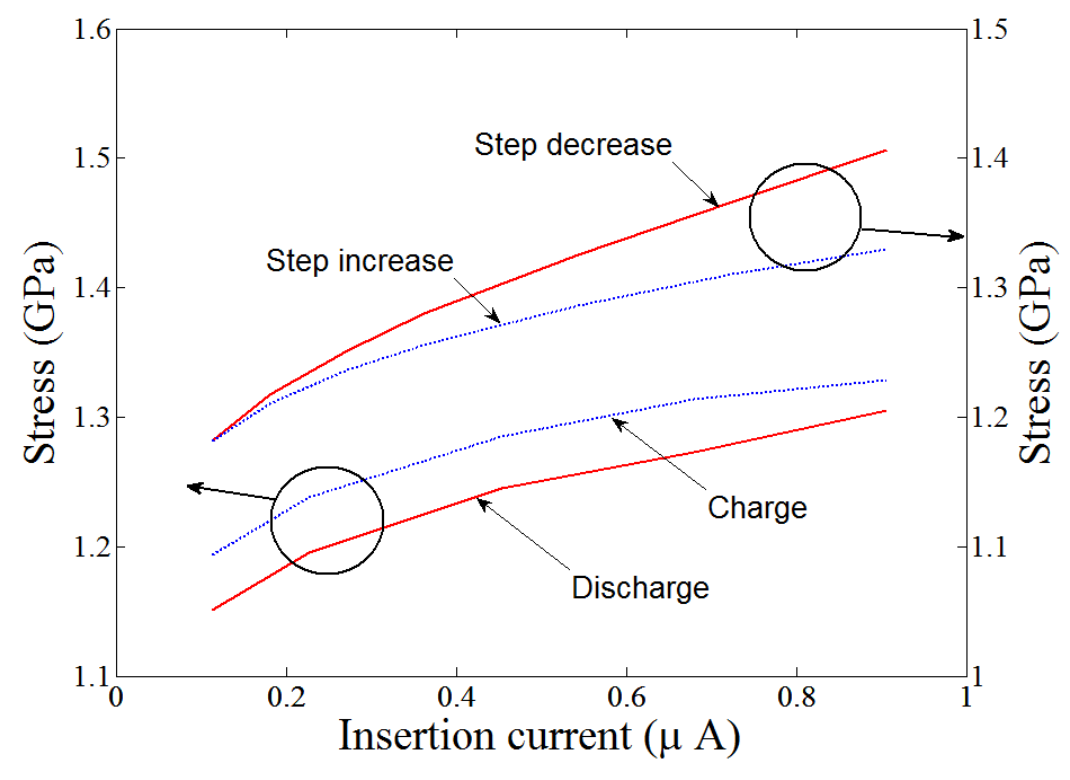

Fig. 7: Predicted variation of flow stress magnitude with Li insertion current, re-plotted from Fig 6. 


\begin{tabular}{|l|l|}
\hline Molar density of pure Si $c_{S i 0}$ & $78740 \mathrm{~mol} \mathrm{~m}^{-3}$ \\
\hline Initial molar density of $\mathrm{Li} c_{L i 0}$ & 0 \\
\hline Initial concentration of unoccupied Si sites $c_{V 0}$ & Variable \\
\hline Molar volume of unoccupied Si sites $\Omega_{V}$ & $1 / c_{S i 0}$ \\
\hline Molar volume of Li $\Omega_{L i}$ & $0.7 / c_{S i 0}$ \\
\cline { 2 - 2 } Maximum Li atoms per Si atom $r$ & 3.75 \\
\hline Fraction of Li occupying vacant Si sites $\beta$ & Variable \\
\hline Gas constant $R$ & $8.314 \mathrm{~J} \mathrm{~mol}^{-1} \mathrm{~K}^{-1}$ \\
\hline Temperature $T$ & $298 \mathrm{~K}$ \\
\hline Faraday constant $F$ & $96485 \mathrm{C} \mathrm{mol}^{-1}$ \\
\hline Biaxial modulus of Li-Si $(1-v) /(2 \mu(1+v))$ & $100 \mathrm{GN} \mathrm{m}$ \\
\hline Characteristic strain rate $\dot{\varepsilon}_{0}$ & $1 \mathrm{~s}^{-1}$ \\
\hline Flow stress $\sigma_{0}$ & $2.25 \mathrm{GPa}$ \\
\hline Stress exponent $m$ & 20 \\
\hline Rate of Si vacancy nucleation with plastic strain $v_{0}^{p}$ & Variable \\
\hline Rate of thermal Si vacancy nucleation $v_{0}^{T}$ & Variable \\
\hline Exponent for thermal vacancy nucleation $n$ & 1 \\
\hline Free energy coefficient $\Lambda_{1} / F$ & $-0.74 \mathrm{~V}$ \\
\hline Free energy coefficient $\Lambda_{2} / F$ & $0.8735 \mathrm{~V}$ \\
\hline Free energy coefficient $\Lambda_{3} / F$ & $0.7185 \mathrm{~V}$ \\
\hline Free energy coefficient $\Lambda_{4} / F$ & $-4.504 \mathrm{~V}$ \\
\hline Free energy coefficient $\Lambda_{5} / F$ & $6.876 \mathrm{~V}$ \\
\hline Free energy coefficient $\Lambda_{6} / F$ & $-4.6272 \mathrm{~V}$ \\
\hline Free energy coefficient $\Lambda_{7} / F$ & $1.1744 \mathrm{~V}$ \\
\hline Initial film thickness $h_{0}$ & $100 \mathrm{~nm}$ \\
\hline Li insertion current & $8.75 \mu A$ \\
\hline
\end{tabular}

Table 1: List of parameter values used to model cyclic lithiation of an amorphous thin film Si electrode 